\title{
Stable Neo-Hookean Flesh Simulation
}

\author{
BREANNAN SMITH, FERNANDO DE GOES, and THEODORE KIM, Pixar Animation Studios
}

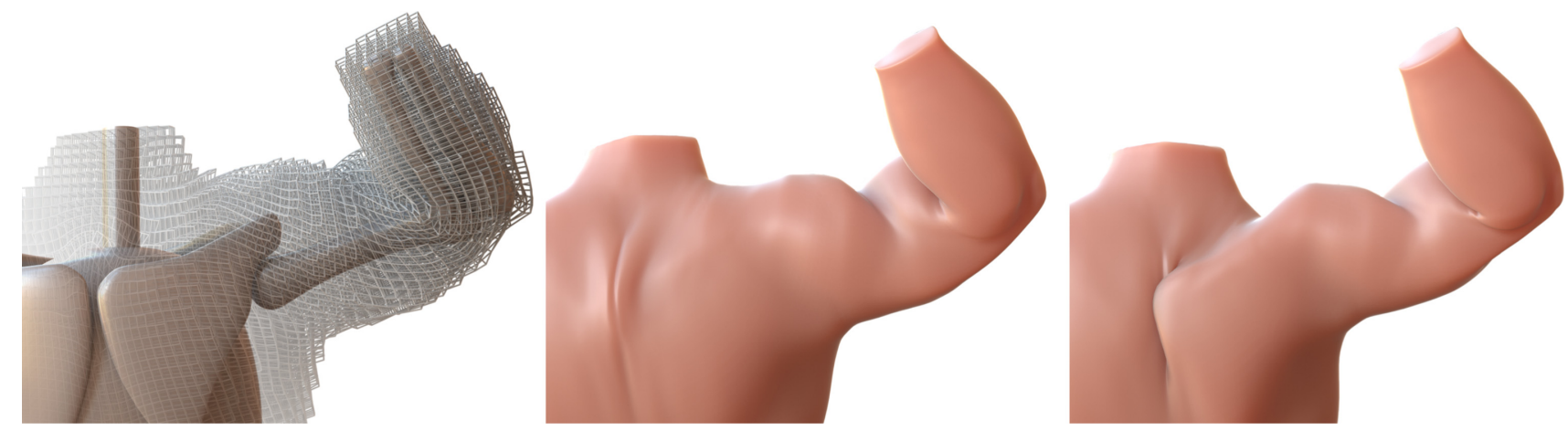

Fig. 1. Left: Thirteen skeletal bones drive a hexahedral lattice with 45,809 elements and 156,078 degrees of freedom. Center: A quasi-static simulation with our new Neo-Hookean model and a Poisson's ratio of $v=0.488$. Wrinkles and bulges emerge from our model's excellent volume-preserving properties. An average time step with our model took 13.7 Newton iterations, 5,860 Conjugate Gradient (CG) iterations, and 25.6 seconds. Right: The same simulation with corotational elasticity and $v=0.488$. The model fails to preserve volume and instead collapses the trapezius and forms a spurious fold around the shoulder blade. The artifacts persist across all values of $v$. An average time step with this model took 17.9 Newton iterations, 16,183 CG iterations, and 46.6 seconds. @Disney/Pixar.

Nonlinear hyperelastic energies play a key role in capturing the fleshy appearance of virtual characters. Real-world, volume-preserving biological tissues have Poisson's ratios near $1 / 2$, but numerical simulation within this regime is notoriously challenging. In order to robustly capture these visual characteristics, we present a novel version of Neo-Hookean elasticity. Our model maintains the fleshy appearance of the Neo-Hookean model, exhibits superior volume preservation, and is robust to extreme kinematic rotations and inversions. We obtain closed-form expressions for the eigenvalues and eigenvectors of all of the system's components, which allows us to directly project the Hessian to semipositive definiteness, and also leads to insights into the numerical behavior of the material. These findings also inform the design of more sophisticated hyperelastic models, which we explore by applying our analysis to Fung and Arruda-Boyce elasticity. We provide extensive comparisons against existing material models.

CCS Concepts: • Computing methodologies $\rightarrow$ Physical simulation;

Additional Key Words and Phrases: Physically-based simulation, elasticity

\section{ACM Reference format:}

Breannan Smith, Fernando de Goes, and Theodore Kim. 2018. Stable NeoHookean Flesh Simulation. ACM Trans. Graph. 37, 2, Article 12 (March 2018), 15 pages.

https://doi.org/10.1145/3180491

Authors' addresses: B. Smith, F. de Goes, and T. Kim, 1200 Park Avenue, Emeryville, CA 94608; emails: \{breannan, fernando, tkim\}@pixar.com.

Permission to make digital or hard copies of all or part of this work for personal or classroom use is granted without fee provided that copies are not made or distributed for profit or commercial advantage and that copies bear this notice and the full citation on the first page. Copyrights for components of this work owned by others than the author(s) must be honored. Abstracting with credit is permitted. To copy otherwise, or republish, to post on servers or to redistribute to lists, requires prior specific permission and/or a fee. Request permissions from permissions@acm.org.

2018 Copyright is held by the owner/author(s). Publication rights licensed to ACM.

ACM 0730-0301/2018/03-ART12 \$15.00

https://doi.org/10.1145/3180491

\section{INTRODUCTION}

The elastic energy used to model deformations determines the visual quality of a simulation, so it must be chosen carefully for virtual characters and virtual humans. The defining quality of biological tissues such as muscle and fat is volume preservation, which is reflected in their high Poisson's ratios $v \in[0.45,0.5)$ (Greaves et al. 2011) and the corresponding visual features that emerge (Figure 2). However, this near-incompressible regime is notoriously difficult to simulate robustly and accurately. For instance, the popular corotational model for elasticity (Chao et al. 2010; McAdams et al. 2011; Müller et al. 2002; Nesme et al. 2009) ostensibly supports materials within this range, but it linearizes the volume term in a way that compromises both its volumetric properties and its visual results (Figure 1, right). Alternatively, force-filtering approaches (Irving et al. 2004; Teran et al. 2005) inherit the visual quality of the NeoHookean model but introduce additional user parameters.

In this work, we propose a novel Neo-Hookean energy that retains the rich, volumetric character of biological materials and does not need any filter parameters. While motivated by biological tissues where $v \approx 0.5$, our model behaves well across a wide range of Poisson's ratios $(v \in[0,0.5))$. Our model exhibits better volume preservation than equivalent filtered models and does not break down under the large kinematic rotations that often occur in quasistatic simulations. The computational performance of our method is competitive with, and often better than, existing methods. We know of no other model that possesses all of these advantages.

We achieve these properties by performing an analysis that completely characterizes the Hessian of the energy and results in closed-form expressions for the eigenvectors and eigenvalues of each of its terms. The eigendecomposition of the overall system can then be written as a set of compact expressions, which we 

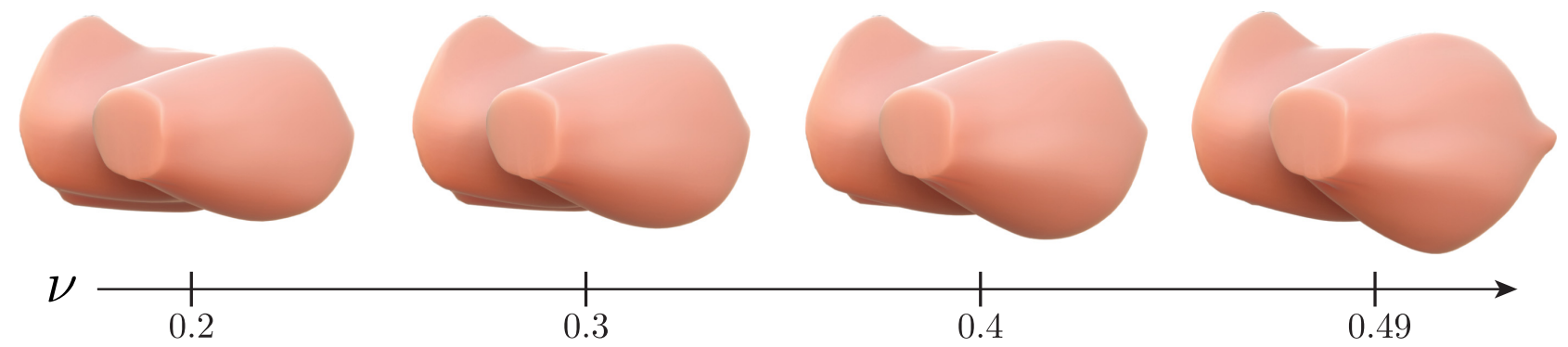

0.3

0.4

0.49

Fig. 2. Volume preservation is the key to convincing flesh simulations. We simulate an elbow flexion over a range of Poisson's ratios $v$ with our stable Neo-Hookean model. Smaller values of $v$ resemble a skinning, and it is not until $v \approx 1 / 2$ that we obtain significant volumetric bulging in the forearm. (-Disney/Pixar.

use to isolate the sources of indefiniteness and project them back to semidefiniteness. As a consequence, we can safely apply our energy to Newton-type implicit integration schemes that use conjugate gradient-based linear solvers. While this analysis was motivated by our Neo-Hookean model, it is sufficiently generic that it also supports any model formulated in terms of the first and third invariants of the deformation gradient. As preliminary examples, we show how it can be extended to Fung (2013) and Arruda-Boyce (1993) elasticity. We conclude with detailed comparisons to existing hyperelastic energies. In particular, we are able to phrase the popular corotational model as a linearization of the Neo-Hookean energy (Section 5.1) and position our model on a spectrum of successive approximations to this energy.

\section{BACKGROUND}

Before presenting our material model, we provide a brief review of existing hyperelastic energies and numerical simulators. In the following, we represent scalars as unbolded letters $(x, J)$, vectors as bold lowercase letters $(\mathbf{x})$, and matrices as bold uppercase letters $(\mathrm{X})$. Greek letters $(\Psi, \alpha)$ represent scalars.

\subsection{Deformation Gradient}

The fundamental measure of nonlinear deformation is the deformation gradient F. Here, we label its columns with vectors and its entries with scalars:

$$
\mathbf{F}=\left[\begin{array}{l|l|l}
\mathbf{f}_{0} & \mathbf{f}_{1} & \mathbf{f}_{2}
\end{array}\right]=\left[\begin{array}{lll}
f_{0} & f_{3} & f_{6} \\
f_{1} & f_{4} & f_{7} \\
f_{2} & f_{5} & f_{8}
\end{array}\right] .
$$

Table 1 summarizes the derived quantities of $F$ we will use. One factorization requires special attention: the rotation variant SVD (Higham 2008; Irving et al. 2004; Twigg and Kačić-Alesić 2010) of F, which we write as

$$
\mathbf{F}=\mathbf{U} \Sigma \mathbf{V}^{T} \text { and } \quad \Sigma=\left[\begin{array}{rrr}
\sigma_{0} & 0 & 0 \\
0 & \sigma_{1} & 0 \\
0 & 0 & \sigma_{2}
\end{array}\right]
$$

Unlike the standard SVD convention, the rotation variant moves reflections to $\Sigma$, so $\mathbf{U}$ and $\mathbf{V}$ are rotations with $\operatorname{det}(\mathbf{U})=\operatorname{det}(\mathbf{V})=1$, and $\Sigma$ is allowed to have a negative entry.

\subsection{Hyperelastic Energies}

The elastic behavior of a deformable body can be specified in terms of a hyperelastic energy density $\Psi$, such as the corotational (CR)
Table 1. Quantities Derived from the Deformation Gradient F

\begin{tabular}{ll}
\hline Symbol & \multicolumn{1}{c}{ Definition } \\
\hline $\mathrm{F}=\mathrm{RS}$ & Polar decomposition \\
$J=\operatorname{det}(\mathrm{F})$ & Relative volume change \\
$\mathrm{C}=\mathrm{F}^{T} \mathrm{~F}$ & Right Cauchy-Green tensor \\
$I_{C}=\operatorname{tr}(\mathrm{C})$ & First right Cauchy-Green invariant \\
\hline
\end{tabular}

material (McAdams et al. 2011):

$$
\Psi_{\mathrm{CR}}=\mu\|\mathrm{F}-\mathrm{R}\|_{F}^{2}+\frac{\lambda}{2} \operatorname{tr}^{2}(\mathrm{~S}-\mathrm{I}) .
$$

Here, $\mu$ and $\lambda$ are the Lamé constants, $\mathbf{R}$ and $\mathbf{S}$ form the polar decomposition of $\mathbf{F}$ (Table 1 ), and $\mathbf{I}$ is a $3 \times 3$ identity matrix. Forces can be derived from this energy by several means, but a popular approach uses the first Piola-Kirchhoff (PK1) stress tensor, which we denote $\mathrm{P}(\mathrm{F})$. The $\mathrm{PK} 1$ for the corotational material is

$$
\mathbf{P}_{\mathrm{CR}}(\mathbf{F})=\frac{\partial \Psi}{\partial \mathbf{F}}=\mathbf{R}[\mu(\mathrm{S}-\mathbf{I})+\lambda \operatorname{tr}(\mathrm{S}-\mathbf{I}) \mathbf{I}]
$$

After integrating over a volume $V$ and changing variables to vertex displacements $\mathbf{u}$, we obtain a matrix, $V \mathbf{P}(\mathbf{F}) \frac{\partial \mathrm{F}}{\partial \mathbf{u}}$, whose columns are vertex forces. The term $\frac{\partial \mathrm{F}}{\partial \mathrm{u}}$ varies based on the element type, e.g., tetrahedra or hexahedra, and basis type, e.g., linear or quadratic (Bargteil and Cohen 2014). Other texts describe this derivation (Müller et al. 2008; Sifakis and Barbic 2012), so we will deal directly with $\Psi$ and $\mathbf{P}(\mathbf{F})$ and defer to these references for further details.

\subsection{Robustness Challenges}

A key drawback of nonlinear hyperelastic energies is that they usually contain singularities. Early work on deformable simulation (Hirota et al. 2001) detected these "illegal" states and carefully avoided them using a backtracking line search. These searches are challenging to perform robustly, because a $\mathrm{NaN}$ in a single degree of freedom can quickly grow to pollute larger regions of the simulation. This backtracking approach also precludes simulations where the solution contains inverted elements, which often occurs in production environments.

Irving et al. (2004) described an explicit method for simulating nonlinear materials that instead used the rotation variant SVD (Equation (2)) to write a filtered stress tensor $\mathbf{P}(\Sigma)$ defined in F's principal stretch space $\Sigma$. For specific values of $\Sigma$ where $\mathrm{P}(\Sigma)$ was known to behave badly, the tensor was clamped to constant 
or linear functions. Thus, no backtracking was needed to avoid illegal states. In order to use this method, the user identifies badly behaved regions of $\mathbf{P}(\Sigma)$ and sets a filtering threshold. Notably, the usual convention for the rotation variant SVD is to push reflections into the smallest singular value, but subsequent work has shown that this can introduce subtle problems, including discontinuities and nonoptimal inversion recovery directions (Georgii and Westermann 2008; Schmedding and Teschner 2008). The work of Civit-Flores and Susín (2014) provides an excellent overview.

Implicit integration of the force-filtering approach was described by Teran et al. (2005). Since the fourth-order tensor $\frac{\partial \mathrm{P}(\mathrm{F})}{\partial \mathrm{F}}$ may become indefinite, a separate derivation was needed to guarantee its positive definiteness. The tensor was diagonalized into one $3 \times 3$ and three $2 \times 2$ matrices, and eigenvalues were clamped to a threshold. This approach has the limitation that $\Psi, P(F)$ and $\frac{\partial \mathrm{P}(\mathrm{F})}{\partial \mathrm{F}}$ can drift out of sync. This issue was alleviated by Stomakhin et al. (2012) by applying the filter directly to $\Psi$. Xu et al. (2015) proposed an alternative where users design force curves in principal stretch space. We instead present an energy that is agnostic to any reflection convention, even in the presence of inverted elements.

Alternative deformation schemes such as position-based dynamics (Bender et al. 2015; Umetani et al. 2014) and projective dynamics (Bouaziz et al. 2014) have recently emerged. One advantage of these schemes is that the Hessian $\frac{\partial \mathrm{P}(\mathrm{F})}{\partial \mathrm{F}}$ does not need to be constructed explicitly. However, projective dynamics still need to specify filtering thresholds and reflection conventions (Narain et al. 2016; Wang and Yang 2016) to avoid numerical singularities. The advantage that our model requires neither carries over directly.

\section{ENERGY FORMULATION}

Following the preceding discussion, we design a new hyperelastic energy that is stable in four important ways:

- Inversion stability: The energy is singularity-free and does not need any filters or thresholds.

- Reflection stability: The energy is well behaved regardless of the reflection convention used in the SVD.

- Rest stability: Under zero load, an element's rest shape is preserved.

- Meta-stability under degeneracy: Under point, line, and plane degeneracies, the forces are defined up to rotation.

We start by examining existing hyperelastic energies that go by the name "Neo-Hookean."

\subsection{Existing Neo-Hookean Energies}

The most common version of Neo-Hookean elasticity is (see, e.g., Bonet and Wood (2008))

$$
\Psi_{\mathrm{Neo}}=\frac{\mu}{2}\left(I_{C}-3\right)-\mu \log J+\frac{\lambda}{2}(\log J)^{2} .
$$

Here, $I_{C}$ and $J$ are defined in Table 1 . However, many other versions appear in the literature:

$$
\begin{array}{ll}
\Psi_{\mathrm{A}}=\frac{\mu}{2}\left(I_{C}-3\right)-\mu \log J+\frac{\lambda}{2}(J-1)^{2} & \text { [Ogden 1997], } \\
\Psi_{\mathrm{B}}=\frac{\mu}{2}\left(J^{-2 / 3} I_{C}-3\right)+\frac{\lambda}{2}(J-1)^{2} & \text { [Bower 2009], }
\end{array}
$$

$$
\Psi_{\mathrm{C}}=\frac{\mu}{2}\left(J^{-2 / 3} I_{C}-3\right)+\frac{\lambda}{2}(J-1) \quad \text { [Wang and Yang 2016]. }
$$

We examine these energies under the Valanis-Landel hypothesis (Xu et al. 2015), which posits that many hyperelastic energies can be separated into length (1D), area (2D), and volume (3D) components. The above models contain length and volume terms, but no area terms. Adding an area term yields a Mooney-Rivlin model.

1D Length Term: Mooney (1940) originally proposed the energy

$$
\Psi_{\mathrm{M}}=\frac{\mu}{2}\left(I_{C}-3\right),
$$

which was later dubbed the "Neo-Hookean" energy by Rivlin (1948). When unconstrained, this energy achieves its minimum when the element has collapsed to zero volume, i.e., when $I_{C}=0$ and $\Psi_{M}=-3$. Mooney additionally imposed the hard constraint that $J=1$, so the energy is instead minimized at the volumepreserving configuration that is the closest to the stretch space origin. Even without this constraint, we note that $\Psi_{M}$ is well behaved under inversion. The energy relative to a zero-volume configuration is always well defined irrespective of an element's current state.

In contrast, the $\Psi_{\mathrm{B}}$ and $\Psi_{\mathrm{C}}$ energies use a modified Neo-Hookean term $\mu / 2\left(J^{-2 / 3} I_{C}-3\right)$ (Rivlin 1948). The $J^{-2 / 3}$ term factors off the nearest squared isotropic stretch from $I_{C}$, leaving only the squared distortional part of the deformation. It also introduces numerical problems by growing without bound under compression, i.e., as $J \rightarrow 0$, and becoming undefined at $J=0$. Therefore, we prefer to use a $\Psi_{M}$ length term in lieu of a modified energy.

3D Volume Term: The remaining terms in the above energies are volume-preserving penalty terms. The volume terms from $\Psi_{\mathrm{Neo}}$ clearly present numerical difficulties:

$$
\Psi_{\mathrm{Neo}, \text { volume }}=-\mu \log J+\frac{\lambda}{2}(\log J)^{2} .
$$

In addition to growing unbounded as $J \rightarrow 0$, they become infeasible for $J<0$. These difficulties motivated the design of the forcefiltering approach (Irving et al. 2004). Any energy with a logarithm, including $\Psi_{\mathrm{A} \text {, volume }}=-\mu \log J+\lambda / 2(J-1)^{2}$, will also have these problems.

In contrast, $\Psi_{\mathrm{B} \text {, volume }}=\lambda / 2(J-1)^{2}$ does not have these issues and is bounded, well defined, and invertible. Such terms have been used (Martin et al. 2011; Teschner et al. 2004) to sidestep the need for any inversion handling. A promising candidate is then

$$
\Psi_{\mathrm{D}}=\frac{\mu}{2}\left(I_{C}-3\right)+\frac{\lambda}{2}(J-1)^{2}
$$

but it has a key drawback that we must address.

\subsection{Rest Stabilization}

While $\Psi_{D}$ possesses inversion stability, it lacks what we will call rest stability. In the solid mechanics literature (e.g., Bonet and Wood (2008), Section 6.4.3), it is often mentioned that the hyperelastic energy should vanish at identity. This occurs with $\Psi_{\mathrm{D}}$, but it is more important that the PK1 resolve to zero, as this is the true indicator that the energy has been extremized. Otherwise, forces appear when the body is at rest and overwrite the intended rest state with a different, parameter-dependent state. This is a known 
side effect of certain barrier functions (Schüller et al. 2013). One solution is to increase the volume penalty constant $\lambda$ dramatically in order to reduce the visual appearance of the artifact (e.g., Blemker et al. (2005) suggests $\sim 1,000 \mu$ ). We avoid this approach, because it precludes the artifact-free simulation of materials with lower Poisson's ratios. Scaling $\lambda$ also increases the indefiniteness of the Hessian (see Section 4.6) and should be done judiciously.

Intuitively, the problem is that the effective rest state no longer coincides with $J=1$. Instead, it coincides with a smaller $J$ that causes an element to slightly deflate. Consequently, we ask whether we can inflate each element so that, when it deflates, it settles to rest at $J=1$. To answer this question, we consider the PK1 of $\Psi_{D}$ :

$$
\mathbf{P}_{\mathrm{D}}(\mathbf{F})=\mu \mathbf{F}+\lambda \frac{\partial J}{\partial \mathbf{F}}(J-1) .
$$

An energy is rest stable if $P_{D}(I)=0$ but $P_{D}(I)$ is nonzero:

$$
\mathbf{P}_{\mathrm{D}}(\mathbf{I})=\mu \mathbf{I}+\lambda \frac{\partial \operatorname{det}(\mathbf{I})}{\partial \mathbf{F}}(\operatorname{det}(\mathbf{I})-1)=\mu \mathbf{I}
$$

To recover rest stability, we modify $(J-1)^{2}$ to shift the root from 1 to $\alpha$; i.e., we inflate the element to a volume greater than one:

$$
\left\{\begin{aligned}
\Psi_{\mathrm{E}} & =\frac{\mu}{2}\left(I_{C}-3\right)+\frac{\lambda}{2}(J-\alpha)^{2} \\
\mathbf{P}_{\mathrm{E}}(\mathbf{F}) & =\mu \mathbf{F}+\lambda \frac{\partial J}{\partial \mathbf{F}}(J-\alpha) .
\end{aligned}\right.
$$

Solving for an $\alpha$ that satisfies $\mathbf{P}_{\mathrm{E}}(\mathbf{I})=0$ yields $\alpha=1+\mu / \lambda$ :

$$
\Psi_{\mathrm{E}}=\frac{\mu}{2}\left(I_{C}-3\right)+\frac{\lambda}{2}\left(J-1-\frac{\mu}{\lambda}\right)^{2} .
$$

We can additionally expand the quadratic to obtain

$$
\Psi_{\mathrm{E}}=\frac{\mu}{2}\left(I_{C}-3\right)-\mu(J-1)+\frac{\lambda}{2}(J-1)^{2}+\left(\frac{\mu}{\lambda}\right)^{2} .
$$

Since constants disappear under differentiation, this material model is functionally equivalent to

$$
\Psi_{\mathrm{E}}=\frac{\mu}{2}\left(I_{C}-3\right)-\mu(J-1)+\frac{\lambda}{2}(J-1)^{2} .
$$

This is strikingly similar to the original $\Psi_{\mathrm{Neo}}$ from Equation (5), except the $\log J$ terms have been replaced with $(J-1)$. We make two important observations. First, $\Psi_{\mathrm{Neo}}$ is rest stable, but not singularity-free. Second, $(J-1)$ is the first term in the Taylor series of $\log J$ at $J=1$. Essentially, we have performed a singularityremoving Taylor truncation of $\Psi_{\mathrm{Neo}}$ about a volume-preserving state, resulting in an inversion and rest-stable material model. We can also verify that our energy $\Psi_{\mathrm{E}}$ is reflection stable. The $I_{C}$ term involves the squared singular values of $\mathrm{F}$, so any negation convention is irrelevant. Since the $J$ term is the product of the singular values, the sign convention is again irrelevant. Similar reasoning can be used for PK1, and we show in Section 4.5.1 that the Hessian is agnostic to the convention as well.

\subsection{Meta-Stability under Degeneracy}

The energy now has inversion, reflection, and rest stability. However, its behavior must be examined under degeneracy, i.e., when an element has been crushed to a plane, line, or point. This examination can also be viewed as a Drucker stability analysis (Bower 2009).

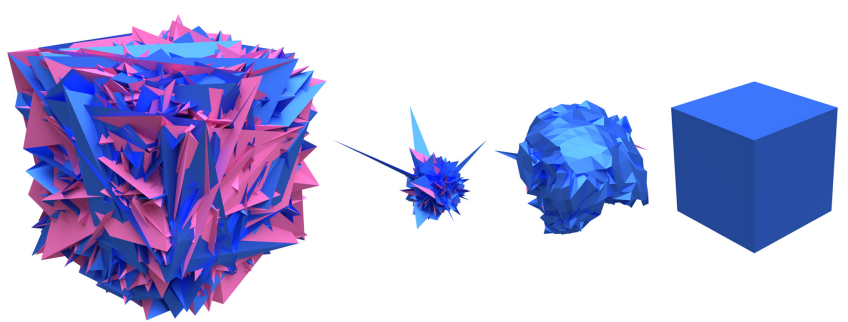

Fig. 3. To stress-test our stable Neo-Hookean $(v=0.49)$ model, we randomly scramble a cube's vertices within a space of twice its volume. The cube recovers its rest pose, highlighting our model's robustness under extreme, inverted configurations.

An extensive treatment of all three degeneracies is given in the supplemental materials, but we will sketch the main results here. For the case where an element is crushed to a plane, the energy is stable. Material forces appear along the normal direction to the plane and work to restore the original shape.

When the element is crushed to a line, the energy is meta-stable. The forces resolve to zero, but the Hessian is negative definite. For a material that is invertible, this meta-stable state is correct. If an unconstrained element has been crushed to exactly a line, the shape it should return to is underdetermined, because the configuration is equidistant from all possible rotations of the original shape about the line. However, negative definiteness guarantees that any perturbation (e.g., momentum) will select a rotation, and the element will self-restore. The alternative to meta-stability is a singularity at this configuration, which is clearly undesirable.

When an element is crushed to a point, i.e., $F=0$, the forces disappear and the Hessian is positive definite, which means the configuration will remain stable even in the presence of perturbations. This behavior is undesirable, though the basin of attraction is vanishingly small for Poisson's ratios that correspond to biological tissue $(v \geq 0.45)$. For completeness, we introduce one more term to eliminate the basin across a wide range of $v$.

We add a regularized origin barrier $\log \left(I_{C}+\delta\right)$ that inserts a peak of negative definiteness about $\mathbf{F}=0$ but uses $\delta$ to smooth away the logarithmic singularity. In the supplemental material, we show that $\delta=1$ is the value that exactly produces semidefiniteness at $\mathbf{F}=0$. Our final energy is then

$$
\Psi_{\text {new }}=\frac{\mu}{2}\left(I_{C}-3\right)+\frac{\lambda}{2}(J-\alpha)^{2}-\frac{\mu}{2} \log \left(I_{C}+1\right) .
$$

As we derive in the supplemental material, the rest-stability term now shifts to $\alpha=1+\mu / \lambda-(\mu / 4) \lambda$.

\subsection{Lamé Reparameterization}

With this energy, we now consider the reparameterization of the material parameters $\mu$ and $\lambda$ in order to maintain consistency with Hooke's law. To this end, we shift the values of $\mu$ and $\lambda$ so that our model reproduces the PK1 of linear elasticity, which is of the form

$$
\mathbf{P}(\mathbf{F})=2 \mu_{\text {Lamé }} \epsilon+\lambda_{\text {Lamé }} \operatorname{tr}(\epsilon) \mathbf{I},
$$

where $\epsilon=1 / 2\left(\mathbf{F}+\mathbf{F}^{T}\right)-\mathbf{I}$ is the linearized strain tensor, and the coefficients $\mu_{\text {Lamé }}$ and $\lambda_{\text {Lamé }}$ are the Lamé parameters in linear elasticity. The linearization of the stress derived from Equation (14) 
becomes consistent with this expression if we set $\mu=4 / 3 \mu_{\text {Lamé }}$ and $\lambda=\lambda_{\text {Lamé }}+5 / 6 \mu_{\text {Lamé }}$. The expression for Poisson's ratio shifts to

$$
v=\frac{\lambda-(5 / 8) \mu}{2(\lambda+(1 / 8) \mu)} .
$$

Note that the same methodology can be applied to any of the previous energies. In particular, the reparameterization of Equation (13) yields $\mu=\mu_{\text {Lamé }} \lambda=\lambda_{\text {Lamé }}+\mu_{\text {Lamé }}$, and $v=(\lambda-\mu) /(2 \lambda)$.

Using this new expression for Poisson's ratio (Equation (16)), we can analyze our energy to confirm that it does not introduce any spurious minima over the range $v \in[0,0.5)$. The details are again given in the supplemental materials, and we will sketch the results here. We are able to show symbolically that the critical points of $\Psi_{\text {new }}$ (including maxima and saddle points) only appear under uniform scaling. Using techniques similar to that employed for metastability, we analyze this deformation mode and find that spurious minima only appear when $\lambda / \mu<0.1525$. This corresponds to the auxetic regime of $v \leq-0.85$, so our model is stable for any $v \in[0,0.5)$.

\section{ENERGY EIGENANALYSIS}

We show that it is possible to perform a complete eigenanalysis of Equation (14). In particular, we construct closed-form eigenvalues and eigenvectors of each of the terms in the Hessian, and obtain a compact expression for the eigendecomposition of their sum. These expressions will be used to construct positive semidefinite versions of the Hessian, and also to develop a qualitative understanding of the energy. We begin by specifying a notation for fourth-order tensors.

\subsection{Tensor Notation}

There are many $n^{\text {th }}$ order tensor notations (e.g. (Kolda and Bader 2009; Simmonds 2012)), but we will specialize to $4^{\text {th }}$ order tensors. Similar to Golub and Van Loan (2012), we define vectorization $\operatorname{vec}(\cdot)$ as column-wise flattening of a matrix into a vector:

$$
\mathbf{A}=\left[\begin{array}{ll}
a & c \\
b & d
\end{array}\right] \quad \operatorname{vec}(\mathbf{A})=\left[\begin{array}{l}
a \\
b \\
c \\
d
\end{array}\right]
$$

Since vec(A) is a vector, we could denote it as a. However, we

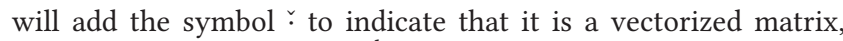
i.e. $\operatorname{vec}(\mathrm{A})=$ ă. We denote $4^{\text {th }}$ order tensors in matrix-of-matrices form using blackboard bold:

$$
\mathbb{A}=\left[\begin{array}{ll}
{\left[\begin{array}{ll}
a & c \\
b & d
\end{array}\right]} & {\left[\begin{array}{ll}
i & k \\
j & l
\end{array}\right]} \\
{\left[\begin{array}{ll}
e & g \\
f & h
\end{array}\right]} & {\left[\begin{array}{ll}
m & o \\
n & p
\end{array}\right]}
\end{array}\right]=\left[\begin{array}{ll}
{\left[\mathrm{A}_{00}\right]} & {\left[\mathrm{A}_{01}\right]} \\
{\left[\mathbf{A}_{10}\right]} & {\left[\mathbf{A}_{11}\right]}
\end{array}\right] .
$$

When $\operatorname{vec}(\cdot)$ is applied, two unfoldings reorder the $4^{\text {th }}$ order tensor into a $2^{\text {nd }}$ order matrix:

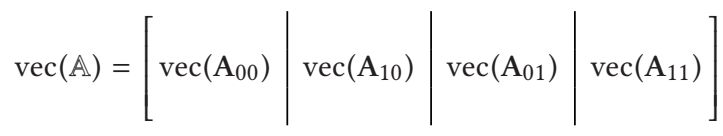

$$
=\left[\begin{array}{llll}
a & e & i & m \\
b & f & j & n \\
c & g & k & o \\
d & h & l & p
\end{array}\right]=\check{\mathrm{A}} .
$$

To emphasize that a matrix is a vectorized ("flattened") tensor, we again use the ‘ notation, as shown in the $\check{A}$ above. This vectorization convention differs from Teran et al. (2005), which clusters the diagonal entries of the submatrices in $\mathbb{A}$. Our convention will allow us to write several expressions in terms of cross products.

\subsection{First Piola-Kirchhoff Stress (PK1)}

We start from the PK1 for Equation (14),

$$
\mathbf{P}(\mathbf{F})=\mu\left(1-\frac{1}{I_{C}+1}\right) \mathbf{F}+\lambda(J-\alpha) \frac{\partial J}{\partial \mathbf{F}},
$$

where $\alpha=1+\mu / \lambda-(\mu / 4) \lambda$. We omit the subscript, as we only consider one model in this section. Using the column-wise notation for $\mathbf{F}$ (Equation (1)) and the identity $J=\mathbf{f}_{0} \cdot\left(\mathbf{f}_{1} \times \mathbf{f}_{2}\right)$, we write $\frac{\partial J}{\partial \mathrm{F}}$ (a.k.a. the cofactor matrix) as cross products:

$$
\frac{\partial J}{\partial \mathbf{F}}=\left[\begin{array}{l|l|l}
\mathbf{f}_{1} \times \mathbf{f}_{2} & \mathbf{f}_{2} \times \mathbf{f}_{0} & \mathbf{f}_{0} \times \mathbf{f}_{1}
\end{array}\right] .
$$

This is a convenient shorthand for computing $\frac{\partial J}{\partial \mathrm{F}}$, and will be useful when analyzing $\frac{\partial^{2} J}{\partial \mathrm{F}^{2}}$.

\subsection{The Energy Hessian Terms}

Using the scalar notation for $\mathbf{F}$ (see Equation (1)), we can write the Hessian of the energy in fourth-order matrix-of-matrices form:

$$
\frac{\partial^{2} \Psi}{\partial \mathbf{F}^{2}}=\frac{\partial \mathbf{P}(\mathbf{F})}{\partial \mathbf{F}}=\left[\begin{array}{l}
{\left[\frac{\partial \mathbf{P}(\mathbf{F})}{\partial f_{0}}\right]\left[\frac{\partial \mathbf{P}(\mathbf{F})}{\partial f_{3}}\right]\left[\frac{\partial \mathbf{P}(\mathbf{F})}{\partial f_{6}}\right]} \\
{\left[\frac{\partial \mathbf{P}(\mathbf{F})}{\partial f_{1}}\right]\left[\frac{\partial \mathbf{P}(\mathbf{F})}{\partial f_{4}}\right]\left[\frac{\partial \mathbf{P}(\mathbf{F})}{\partial f_{7}}\right]} \\
{\left[\frac{\partial \mathbf{P}(\mathbf{F})}{\partial f_{2}}\right]\left[\frac{\partial \mathbf{P}(\mathbf{F})}{\partial f_{5}}\right]\left[\frac{\partial \mathbf{P}(\mathbf{F})}{\partial f_{8}}\right]}
\end{array}\right],
$$

where each entry is defined as

$$
\begin{aligned}
\frac{\partial \mathbf{P}(\mathbf{F})}{\partial f_{i}}= & \underbrace{\mu\left(1-\frac{1}{I_{C}+1}\right) \frac{\partial \mathbf{F}}{\partial f_{i}}}_{\mathbf{T}_{i}}+\underbrace{\mu \frac{2}{\left(I_{C}+1\right)^{2}} \mathbf{F} f_{i}}_{\mathbf{M}_{i}} \\
& +\underbrace{\lambda \frac{\partial J}{\partial \mathbf{F}} \frac{\partial J}{\partial f_{i}}}_{\mathbf{G}_{i}}+\underbrace{\lambda(J-\alpha) \frac{\partial^{2} J}{\partial \mathbf{F} \partial f_{i}}}_{\mathbf{H}_{i}},
\end{aligned}
$$

and each $\frac{\partial \mathbf{P}(\mathbf{F})}{\partial f_{i}} \in \mathbb{R}^{3 \times 3}$. Since we will examine each of the terms in detail, we have tagged them as $\mathbf{T}_{i}, \mathbf{M}_{i}, \mathrm{G}_{i}$, and $\mathbf{H}_{i}$, respectively the Tikhonov, $\mathbf{M u}$, volume Gradient, and volume Hessian terms. The complete Hessian is then their sum:

$$
\begin{aligned}
\frac{\partial^{2} \Psi}{\partial \mathbf{F}^{2}}= & \mu\left(1-\frac{1}{I_{C}+1}\right) \mathbb{T}+\mu \frac{2}{\left(I_{C}+1\right)^{2}} \mathbb{M}(\mathbf{F}) \\
& +\lambda G(\mathbf{F})+\lambda(J-\alpha) \mathbb{H}(\mathbf{F}) .
\end{aligned}
$$

We will examine the eigensystem of each term, and then build the system that results from their sum. 


\subsection{The Tikhonov, Mu, and Gradient Terms}

We start with the Tikhonov term. Since $\frac{\partial \mathrm{F}}{\partial f_{i}}$ is zero except in the $i$ th entry, this becomes an identity matrix under vectorization:

$$
\operatorname{vec}(\mathbb{T})=\check{\mathbf{T}}=\mathbf{I},
$$

where I denotes a $9 \times 9$ identity matrix. This matrix is full rank, positive definite, and independent of the values in $\mathbf{F}$, and serves as a diagonal (Tikhonov) regularizer for the rest of the energy.

For the $\mathrm{Mu}$ term, we vectorize $\mathrm{F}$ into a nine-vector, $\operatorname{vec}(\mathbf{F})=\check{\mathbf{f}}$, which allows the tensor to be written as an outer product:

$$
\operatorname{vec}(\mathbb{M})=\check{\mathbf{M}}=\check{\mathbf{f}}^{T} \text {. }
$$

This rank-one matrix has a single nonzero eigenvalue:

$$
\|\check{\mathbf{f}}\|_{2}^{2} \equiv\|\mathbf{F}\|_{F}^{2} \equiv\left(\sigma_{0}^{2}+\sigma_{1}^{2}+\sigma_{2}^{2}\right),
$$

where $\|\cdot\|_{F}$ denotes the Frobenius norm and $\left\{\sigma_{i}\right\}$ the singular values from Equation (2). The eigenvector is $\breve{f}$ up to normalization: $\check{\mathbf{f}} /\|\check{\mathbf{f}}\|$. The eigenvalue is always nonnegative, and if $\mathbf{F}$ contains a large stretch, the eigenvalue will be large as well.

The gradient term has a similar structure. Using the vectorization $\operatorname{vec}\left(\frac{\partial J}{\partial \mathrm{F}}\right)=\check{\mathrm{g}}$, the tensor becomes an outer product:

$$
\operatorname{vec}(\mathbb{G})=\check{\mathbf{G}}=\check{\mathbf{g}} \check{g}^{T} .
$$

Again, there is a single nonzero, nonnegative eigenvalue:

$$
\|\check{\mathbf{g}}\|_{2}^{2} \equiv\left\|\frac{\partial J}{\partial \mathbf{F}}\right\|_{F}^{2} \equiv\left[\left(\sigma_{0} \sigma_{1}\right)^{2}+\left(\sigma_{0} \sigma_{2}\right)^{2}+\left(\sigma_{1} \sigma_{2}\right)^{2}\right] .
$$

The eigenvector is $\check{\mathbf{g}}$ up to normalization: $\check{\mathbf{g}} /\|\check{\mathbf{g}}\|$.

\subsection{The Volume Hessian}

The $\mathbf{H}_{i}$ term is more involved. Vectorizing $\mathbf{H}_{i}$ reveals the structure

$$
\operatorname{vec}(\mathbb{H})=\check{\mathbf{H}}=\left[\begin{array}{rrr}
\mathbf{0} & -\widehat{\mathbf{f}_{2}} & \widehat{\mathbf{f}_{1}} \\
\widehat{\mathbf{f}_{2}} & \mathbf{0} & -\widehat{\mathbf{f}_{0}} \\
-\widehat{\mathbf{f}_{1}} & \widehat{\mathbf{f}_{0}} & \mathbf{0}
\end{array}\right],
$$

where the symbol $\widehat{r}$ denotes a cross-product matrix:

$$
\widehat{\mathbf{x}}=\left[\begin{array}{rrr}
0 & -x_{2} & x_{1} \\
x_{2} & 0 & -x_{0} \\
-x_{1} & x_{0} & 0
\end{array}\right]
$$

The $\check{\mathbf{H}}$ matrix forms a self-similar cross-product matrix. Equation (28) contains cross-product matrices, and its blocks form a macro-structure that is a cross-product matrix. Similar crossproduct-like structures will continue to appear later.

4.5.1 Volume Hessian Eigenvalues. The eigenvalues of $\mathbf{H}$ can be factored into two characteristic polynomials:

$$
\begin{aligned}
& \varepsilon^{3}-\operatorname{tr}(\mathrm{C}) \varepsilon-2 J=0 \\
& \varepsilon^{3}-\operatorname{tr}(\mathrm{C}) \varepsilon^{2}+\frac{1}{2}\left(\operatorname{tr}^{2}(\mathrm{C})-\operatorname{tr}\left(\mathrm{C}^{2}\right)\right) \varepsilon-\operatorname{det}(\mathrm{C})=0,
\end{aligned}
$$

where $\mathbf{C}$ is from Table 1 , and $\varepsilon$ denotes the eigenvalues of $\check{\mathbf{H}}$. The usual $\lambda$ notation for eigenvalues has been put aside and replaced by $\varepsilon$ because $\lambda$ is already reserved as a material constant.

Equation (31) is simpler to solve, as it corresponds to the characteristic polynomial of $\mathrm{C}$. Given its roots $\left(\varepsilon_{\alpha}, \varepsilon_{\beta}, \varepsilon_{\gamma}\right)$, six of the

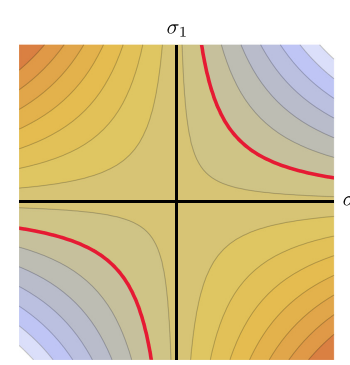

(a) $J=\operatorname{det}(\mathbf{F})$ in $2 \mathrm{D}$

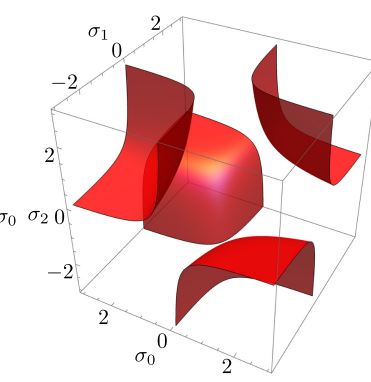

(b) $J=\operatorname{det}(\mathbf{F})$ in $3 \mathrm{D}$
Fig. 4. The set of volume-preserving configurations in $2 \mathrm{D}$ and $3 \mathrm{D}$ stretch space are shown in red. The energy's saddle-point structure is visible in 2D.

eigenvalues of $\check{\mathbf{H}}$ are $\left( \pm \sqrt{\varepsilon_{\alpha}}, \pm \sqrt{\varepsilon_{\beta}}, \pm \sqrt{\varepsilon_{\gamma}}\right)$. The square roots of eigenvalues can be restated using the singular values of $F$ :

$$
\begin{array}{ll}
\varepsilon_{3}=\sigma_{0} & \varepsilon_{6}=-\sigma_{0} \\
\varepsilon_{4}=\sigma_{1} & \varepsilon_{7}=-\sigma_{1} \\
\varepsilon_{5}=\sigma_{2} & \varepsilon_{8}=-\sigma_{2} .
\end{array}
$$

If the SVD of $\mathbf{F}$ has been computed, then six of the eigenvalues of H are already known.

For the remaining three eigenvalues, Equation (30) is a depressed cubic whose roots can be obtained directly:

$$
\varepsilon_{k}=2 \sqrt{\frac{I_{C}}{3}} \cos \left[\frac{1}{3}\left(\arccos \left(\frac{3 J}{I_{C}} \sqrt{\frac{3}{I_{C}}}\right)+2 \pi k\right)\right] \quad k=0,1,2 .
$$

We now have all of the eigenvalues of $\check{\mathbf{H}}$. The negations in Equation (32) and the $I_{C}$ and $J$ terms in Equation (33) render the reflection convention applied to the singular values irrelevant.

There are always negative eigenvalues in the volume Hessian. Three of the six values $\varepsilon_{3} \ldots 8$ are less than or equal to zero, and the cosine function in $\varepsilon_{0} \ldots 2$ guarantees that another one or two eigenvalues are negative. Moreover, we have determined that the other eigenvalues in $\frac{\partial^{2} \Psi}{\partial \mathrm{F}^{2}}$ are nonnegative, so the volume Hessian is the only possible source of negative eigenvalues.

We investigate further by examining the geometry of $J=\operatorname{det}(\mathbf{F})$ in principal stretch space (Ogden 1997; Stomakhin et al. 2012; Xu et al. 2015). The 2D case is easier to visualize, and the intuition carries over to 3D. As shown in red (Figure 4(a)), the set of volumepreserving configurations forms two distinct, disconnected sets. This reflects the nonconvexity of $J=\operatorname{det}(\mathrm{F})$ (Liu et al. 2016), and its saddle-point structure is visible in the contours. The red curve closely resembles the "primary contour" of Stomakhin et al. (2012).

The red points are energetically equivalent, so points outside this curve have no preferred Newton direction, even when the energy is squared, e.g., $(J-1)^{2}$. Additional regularization is needed to resolve this ambiguity, which is the role played by the other terms in $\frac{\partial^{2} \Psi}{\partial \mathrm{F}^{2}}$. The situation worsens in $3 \mathrm{D}$, as the set of volumepreserving configurations forms four components (Figure 4(b)). The intuition remains that saddle points exist everywhere and need to be regularized.

The structure we see in Equations (32) and (33) extends the understanding of the findings in Teran et al. (2005). There, they factor 
the Hessian into one $3 \times 3$ and three $2 \times 2$ eigensystems. This corresponds exactly to the three entangled roots we found for Equation (33) and the three pair-wise roots we found for Equation (32). This underscores the fact that our current analysis can be applied to any system that uses $J=\operatorname{det}(\mathbf{F})$ and is not specific to the energy $\Psi_{\text {volume }}=(J-1)^{2}$.

4.5.2 Volume Hessian Eigenvectors. The eigenvectors of $\mathbf{H}$ can be written in terms of $\check{Q}$ from the eigendecomposition $\check{\mathbf{H}}=\check{\mathrm{Q}} \Lambda \check{Q}^{T}$. However, we prefer the tensor form:

$$
\mathbb{Q}=\left[\begin{array}{lll}
{\left[\mathrm{Q}_{0}\right]} & {\left[\mathrm{Q}_{3}\right]} & {\left[\mathrm{Q}_{6}\right]} \\
{\left[\mathbf{Q}_{1}\right]} & {\left[\mathrm{Q}_{4}\right]} & {\left[\mathrm{Q}_{7}\right]} \\
{\left[\mathrm{Q}_{2}\right]} & {\left[\mathrm{Q}_{5}\right]} & {\left[\mathrm{Q}_{8}\right]}
\end{array}\right]
$$

Each eigenvector is then a $3 \times 3$ matrix instead of a nine-vector.

We start with the eigenvectors corresponding to Equation (31). The eigenvector corresponding to $\varepsilon_{3}$ yields an elegant structure:

$$
\begin{gathered}
\mathrm{Q}_{3}=\frac{1}{\sqrt{2}} \mathrm{UD}_{3} \mathrm{~V}^{T} \\
\mathrm{D}_{3}=\left[\begin{array}{rrr}
0 & 0 & 0 \\
0 & 0 & 1 \\
0 & -1 & 0
\end{array}\right],
\end{gathered}
$$

where $\mathbf{U}$ and $\mathbf{V}$ are from the SVD of $\mathbf{F}$ (Equation (2)) and the $1 / \sqrt{2}$ is a normalization factor. This eigenvector has a "pseudocross-product" structure that we have not seen anywhere else. The middle matrix $D_{3}$ is a cross-product matrix for the basis vector $\mathbf{e}_{0}=\left[\begin{array}{lll}-1 & 0 & 0\end{array}\right]^{T}$. In fact, for the special case where $\mathbf{U}=\mathbf{V}$, Equation (35) becomes a standard cross-product on the first column of U, i.e., $Q_{3}=1 / \sqrt{2} \widehat{\mathbf{u}}_{0}$. Equation (35) has a more general form where $\mathrm{U}$ and $\mathrm{V}$ differ, and corresponds to an infinitesimal rotation along the $\sigma_{0}$ axis in stretch space.

The complementary eigenvector for $\varepsilon_{6}$, has a similar structure:

$$
\begin{gathered}
\mathbf{Q}_{6}=\frac{1}{\sqrt{2}} \mathbf{U D}_{6} \mathbf{V}^{T} \\
\mathbf{D}_{6}=\left[\begin{array}{lll}
0 & 0 & 0 \\
0 & 0 & 1 \\
0 & 1 & 0
\end{array}\right] .
\end{gathered}
$$

Note that the negative has been removed from the last row. $\mathbf{D}_{3}$ has been multiplied by a reflection, making $Q_{6}$ a reflected pseudocross-product. This corresponds to an infinitesimal rotation along the antipodal axis to $D_{3}$. The eigenvectors for $Q_{4}, Q_{5}, Q_{7}$, and $Q_{8}$ follow analogously as the cross-products for $\mathbf{e}_{1}=\left[\begin{array}{lll}0 & -1 & 0\end{array}\right]^{T}$ and $\mathbf{e}_{2}=\left[\begin{array}{lll}0 & 0 & -1\end{array}\right]^{T}$. We explicitly list all six eigenvectors in Appen$\operatorname{dix}$ A.

The eigenvectors for the depressed cubic (Equation (30)) are also defined by $\mathrm{U}$ and $\mathrm{V}$ but contain a conventional diagonal:

$$
\begin{aligned}
& \mathbf{Q}_{k}=\frac{1}{q_{k}} \mathbf{U D}_{k} \mathbf{V}^{T} \quad k=0 \ldots 2 \\
& \mathbf{D}_{k}=\left[\begin{array}{rrr}
\sigma_{0} \sigma_{2}+\sigma_{1} \varepsilon_{k} & 0 & 0 \\
0 & \sigma_{1} \sigma_{2}+\sigma_{0} \varepsilon_{k} & 0 \\
0 & 0 & \varepsilon_{k}^{2}-\sigma_{2}^{2}
\end{array}\right],
\end{aligned}
$$

where $q_{k}=\left\|\mathbf{D}_{k}\right\|_{F}$ is a normalization factor. A Mathematica notebook Verify_Eigenvectors.nb that symbolically confirms this eigensystem is included in the supplemental materials.

\subsection{The Complete Eigensystem}

With these closed-form expressions for the individual terms, we can analyze the complete system, which we denote $\check{A}$ for Assembled. The vectorized version of Equation (22) is

$$
\check{\mathbf{A}}=\mu\left(1-\frac{1}{I_{C}+1}\right) \mathbf{I}+\mu \frac{2}{\left(I_{C}+1\right)^{2}} \check{\mathbf{f}} \check{\mathbf{f}}^{T}+\lambda \check{\mathbf{g}} \check{\mathbf{g}}^{T}+\lambda(J-\alpha) \check{\mathbf{H}} .
$$

Deriving expressions for the the eigenvalues of a sum of matrices is generally nontrivial (Knutson and Tao 2001). However, our energy has a special structure that allows us to obtain simple expressions for this system.

Since the $\mu\left(1-\frac{1}{I_{C}+1}\right) \mathbf{I}$ term is diagonal, it has nine identical eigenvalues that can be directly added to the eigenvalues of any existing system. The main difficulty arises from computing the sum $\mu \frac{2}{\left(I_{C}+1\right)^{2}} \check{\mathbf{f}} \check{\mathbf{f}}^{T}+\lambda \check{\mathbf{g}} \check{\mathbf{g}}^{T}+\lambda(J-\alpha) \check{\mathbf{H}}$. However, both $\check{\mathbf{f}}^{T} \check{\mathbf{f}}^{T}$ and $\check{\mathbf{g}} \check{\mathbf{g}}^{T}$ are rank-one matrices, which allows the problem to be viewed as a rank-two update to the eigensystem for $\lambda(J-\alpha) \check{\mathbf{H}}$.

The problem can be simplified using deflation (Bunch et al. 1978). To this end, we show in Appendix B that both $\check{g}$ and $\check{f}$ are orthogonal to the six pseudo-cross-product eigenvectors of $\check{\mathbf{H}}$. As a consequence, the eigenvectors of $\check{\mathbf{H}}$ and their corresponding eigenvalues remain unchanged, and the rank-two update has now been simplified to the effect solely on the depressed cubic (Equations (33) and (39)). The system is sufficiently small that direct symbolic examination becomes tractable. The eigenvalues of the rank-two updated matrix are the roots of

$$
\begin{aligned}
& \bar{\varepsilon}^{3}+c_{2} \bar{\varepsilon}^{2}+c_{1} \bar{\varepsilon}+c_{0}=0 \\
& c_{2}=-\|\grave{\mathrm{g}}\|_{2}^{2} \rho-I_{C} \eta \\
& c_{1}=-(1+2 J \rho) I_{C}-6 J \eta+\left(\|\grave{\mathrm{g}}\|_{2}^{2} I_{C}-9 J^{2}\right) \rho \eta \\
& c_{0}=-(2+3 J \rho) J+\left(I_{C}^{2}-4\|\check{\mathbf{g}}\|_{2}^{2}\right) \eta+2 J\left(I_{C}^{2}-3\|\check{\mathbf{g}}\|_{2}^{2}\right) \rho \eta
\end{aligned}
$$

where $\eta$ and $\rho$ are defined as

$$
\eta=\frac{2 \mu}{\left(I_{C}+1\right)^{2}\left(\lambda(J-1)-\frac{3}{4} \mu\right)} \quad \rho=\frac{\lambda}{\lambda(J-1)-\frac{3}{4} \mu} .
$$

The cubic is no longer depressed, and while we could apply the cubic formula to obtain a closed-form expression, we found that solving for the roots using the Jenkins and Traub (1970) rpoly algorithm was more practical. The form of the eigenvectors remains the same, $\mathbf{Q}_{k}=\frac{1}{q_{k}} \mathbf{U D}_{k} \mathbf{V}^{T}$, but the diagonal entries differ,

$$
\mathbf{D}_{k}=\left[\begin{array}{rrr}
\alpha_{0} & 0 & 0 \\
0 & \alpha_{1} & 0 \\
0 & 0 & \alpha_{2}
\end{array}\right]
$$


and instead take the form

$$
\begin{aligned}
\alpha_{0}= & \bar{\varepsilon}_{k}\left(\sigma_{1}+\sigma_{0} \sigma_{2} \eta+J \sigma_{1} \rho\right) \\
& +\sigma_{0} \sigma_{2}+\sigma_{1}\left(\sigma_{0}^{2}-\sigma_{1}^{2}+\sigma_{2}^{2}\right) \eta+J \sigma_{0} \sigma_{2} \rho \\
& +\sigma_{0}\left(\sigma_{0}^{2}-\sigma_{1}^{2}\right) \sigma_{2}\left(\sigma_{1}^{2}-\sigma_{2}^{2}\right) \rho \eta \\
\alpha_{1}= & \bar{\varepsilon}_{k}\left(\sigma_{0}+\sigma_{1} \sigma_{2} \eta+J \sigma_{0} \rho\right) \\
& +\sigma_{1} \sigma_{2}-\sigma_{0}\left(\sigma_{0}^{2}-\sigma_{1}^{2}-\sigma_{2}^{2}\right) \eta+J \sigma_{1} \sigma_{2} \rho \\
& -\sigma_{1}\left(\sigma_{0}^{2}-\sigma_{1}^{2}\right) \sigma_{2}\left(\sigma_{0}^{2}-\sigma_{2}^{2}\right) \rho \eta \\
\alpha_{2}= & \bar{\varepsilon}_{k}^{2}-\bar{\varepsilon}_{k}\left(\sigma_{0}^{2}+\sigma_{1}^{2}\right)\left(\eta+\sigma_{2}^{2} \rho\right) \\
& -\sigma_{2}^{2}-2 J \eta-2 J \sigma_{2}^{2} \rho+\left(\left(\sigma_{0}^{2}-\sigma_{1}^{2}\right) \sigma_{2}\right)^{2} \rho \eta .
\end{aligned}
$$

Final eigensystem: With these expressions for the rank-two updated system, we can add the $\mu_{T}=\mu\left(1-1 /\left(I_{C}+1\right)\right)$ regularization term and arrive at the final eigenvalues. The first three are

$$
\varepsilon_{k}=\lambda(J-\alpha) \bar{\varepsilon}_{k}+\mu_{T} \quad k=0 \ldots 2,
$$

where $\bar{\varepsilon}_{k}$ are the roots of Equation (42). The other six are

$$
\begin{array}{lr}
\varepsilon_{3}=\lambda(J-\alpha) \sigma_{0}+\mu_{T} & \varepsilon_{6}=-\lambda(J-\alpha) \sigma_{0}+\mu_{T} \\
\varepsilon_{4}=\lambda(J-\alpha) \sigma_{1}+\mu_{T} & \varepsilon_{7}=-\lambda(J-\alpha) \sigma_{1}+\mu_{T} \\
\varepsilon_{5}=\lambda(J-\alpha) \sigma_{2}+\mu_{T} & \varepsilon_{8}=-\lambda(J-\alpha) \sigma_{2}+\mu_{T} .
\end{array}
$$

The first three eigenvectors are specified by Equations (39) and (44), and the last six remain the ones described in Appendix A.

\section{DISCUSSION AND EXTENSION}

In this section, we compare our energy to corotational elasticity and show the generality of our analysis by applying it to Fung and Arruda-Boyce hyperelasticity.

\subsection{Corotational as Linearized Neo-Hookean}

In Section 3.2, we described how our energy could be viewed as a singularity-removing Taylor truncation of the original NeoHookean energy $\Psi_{\mathrm{Neo}}$. In order to establish a common basis for comparison, we show next how linearizing $\Psi_{\mathrm{Neo}}$ yields corotational elasticity to within a constant. Starting from the energy in Equation (5),

$$
\Psi_{\mathrm{Neo}}=\frac{\mu}{2}\left(I_{C}-3\right)-\mu \log J+\frac{\lambda}{2}(\log J)^{2},
$$

we can employ the linearization $\log J \approx \operatorname{tr}(\mathrm{S}-\mathrm{I})$ to establish that

$$
\begin{aligned}
\frac{\mu}{2}\left(I_{C}-3\right)-\mu \log J & \approx \frac{\mu}{2}\|\mathbf{F}-\mathbf{R}\|_{F}^{2}, \\
\frac{\lambda}{2}(\log J)^{2} & \approx \frac{\lambda}{2} \operatorname{tr}^{2}(\mathrm{~S}-\mathbf{I}),
\end{aligned}
$$

and then conclude that (see proof in Appendix C)

$$
\Psi_{\mathrm{CR}}=\frac{\mu}{2}\|\mathbf{F}-\mathbf{R}\|_{F}^{2}+\frac{\lambda}{2} \operatorname{tr}^{2}(\mathrm{~S}-\mathrm{I}) .
$$

Note that $\Psi_{\mathrm{CR}}$ contains a $\mu / 2$ instead of the $\mu$ from Equation (3), because the linearization introduces a factor of two that should be accounted for using a Lamé reparameterization similar to Section 3.4. The constant factor is otherwise irrelevant to our discussion.

\subsection{Comparison to Our Energy}

From the discussions in Section 3.2 and Section 5.1, it is clear that our model applies the approximation $\log J \approx(J-1)$, while the corotational model applies the linearization $\log J \approx \operatorname{tr}(\mathrm{S}-\mathrm{I})$. This is a critical difference, because when the linearization $\operatorname{tr}(\mathrm{S}-\mathrm{I})$ is used, the actual volume $J=\sigma_{0} \sigma_{1} \sigma_{2}$ is no longer computed. Instead, it is assumed that $\left(\sigma_{0} \sigma_{1} \sigma_{2}-1\right) \approx\left(\sigma_{0}+\sigma_{1}+\sigma_{2}-3\right)$, which only holds for small deformations where $\left\{\sigma_{i}\right\}$ are near 1 .

The unacceptable severity of the linearization becomes clear when we visualize its energy in the inset on the right. The volume preservation term $\frac{\lambda}{2} \operatorname{tr}^{2}(S-I)$ is minimized when $\operatorname{tr} \Sigma=3$. The corresponding contour is shown in red and includes inverted configurations, as can be seen when the line strays outside the upper right quadrant.

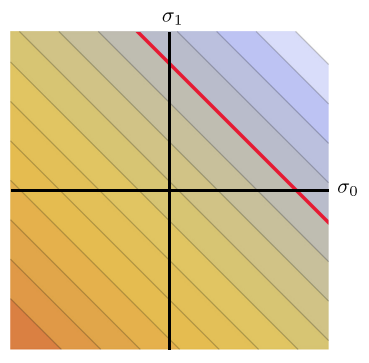
Thus, the energy can report that volume has been perfectly preserved when in fact elements have been crushed to zero volume or inverted. The red contour again resembles Figure 1 from Stomakhin et al. (2012). In that paper, they propose a "fixed" corotational model:

$$
\Psi_{\text {FixCR }}=\mu\|\mathbf{F}-\mathbf{R}\|_{F}^{2}+\frac{\lambda}{2}(J-1)^{2} .
$$

This model is halfway between our model and the original corotational model. As with our model, the $\log J \approx(J-1)$ approximation is applied to the $\frac{\lambda}{2}(\log J)^{2}$ term, but the problematic linearization $\log J \approx \operatorname{tr}(\mathrm{S}-\mathrm{I})$ is still applied to the $\mu \log J$ term. While $\Psi_{\mathrm{FixCR}}$ is more stable than $\Psi_{\mathrm{CR}}$, we show in Figure 5 that it introduces visual artifacts under large deformation.

Finally, we compare to the St. Venant-Kirchhoff (StVK) energy:

$$
\Psi_{\mathrm{StVK}}=\mu\|\mathrm{E}\|_{F}^{2}+\frac{\lambda}{2} \operatorname{tr}^{2}(\mathrm{E}) .
$$

Unlike the corotational energy, the linear $(\mathrm{F}-\mathrm{R})$ has been replaced with a quadratic Green's strain $E=\frac{1}{2}\left(F^{T} \mathbf{F}-\mathbf{I}\right)$. The true volume $J$ is still not computed anywhere, so all of the volume linearization problems from the corotational model are present. Irving et al. (2004) observed that this energy contains spuriously stable inverted rest and flattened states, which makes the standard StVK model unattractive for large deformation. Additional compression resistance terms (Kikuuwe et al. 2009) have been proposed:

$$
\Psi_{\text {StVK, Kikuuwe }}=\mu\|\mathrm{E}\|_{F}^{2}+\frac{\lambda}{2} \operatorname{tr}^{2}(\mathrm{E})+\frac{\kappa}{12}\left(\frac{1-J}{6}\right)^{3},
$$

where the new term is clamped to zero when $J \geq 1$. While this term helps to preserve volume and remove the spurious stable configurations, it deactivates during extension. The artifacts that arise from the linearized volume term then persist in this regime. We show some of these artifacts in Figure 10 and include the model in a more extensive version of Figure 5 in the supplemental material.

\subsection{Extension to Other Energies}

Our eigenanalysis is sufficiently general that it can be used to analyze any strain energy that employs the $I_{C}$ and $J$ strain invariants 


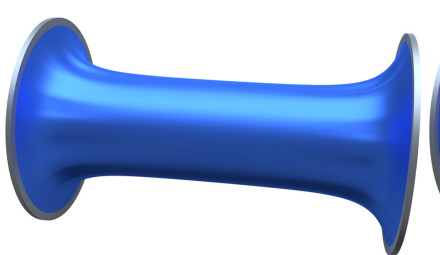

(a) Stable Neo-Hookean

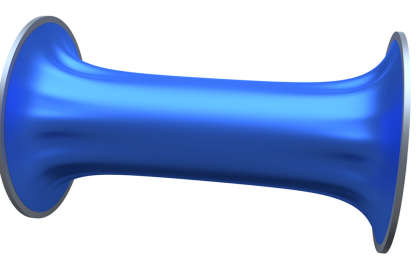

(b) Filtered Neo-Hookean

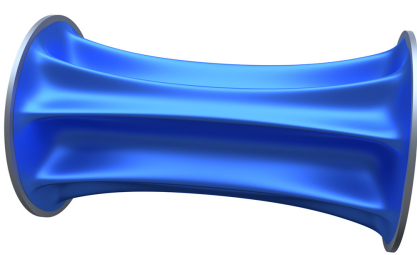

(c) Fixed Co-rotational

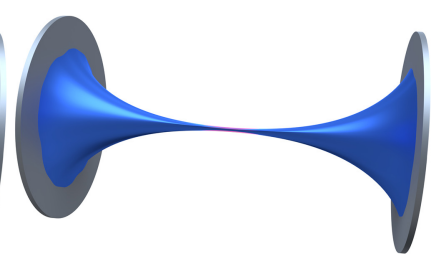

(d) Co-rotational

Fig. 5. A cylinder with 306,406 hexahedra and 965,004 degrees of freedom is stretched by a factor of 3.4 with $v=0.49$. The corotational model fails entirely, and the "fixed" corotational model grows unnatural, sharp fins. Our stable Neo-Hookean model retains the rubber-like appearance of the filtered NeoHookean model, exhibits slightly better volume preservation, and does not require any parameter tuning.

(see Section 7 for comments on $I I_{C}$ ). We have found it straightforward to extend to increased nonlinearities in $I_{C}$.

General Model: Consider a strain energy density expressed in terms of the $I_{C}$ and $J$ invariants that leads to a system of the form:

$$
\check{\mathbf{A}}=\gamma_{I} \mathbf{I}+\gamma_{F} \check{\mathbf{f}}^{T}{ }^{T}+\gamma_{G} \check{\mathbf{g}} \check{g}^{T}+\gamma_{H} \check{\mathbf{H}} \text {. }
$$

The eigensystem can be obtained by setting $\eta=\gamma_{F} / \gamma_{H}$ and $\rho=$ $\gamma_{G} / \gamma_{H}$ in Equation (42), and $\mu_{T}=\gamma_{I}$ and $\lambda(J-\alpha)=\gamma_{H}$ in Equations (45) through (48). We now examine two specific extensions of our analysis.

Fung Hardening: The exponential hardening of Fung-like models (Fung 2013; Pan et al. 2015; Wang and Yang 2016) is a secondary feature of many biological tissues (Kautzman et al. 2012), so we propose a stabilized Fung model:

$$
\Psi_{\text {Fung }}=\frac{\mu_{0}}{2}\left(I_{C}-3\right)+\frac{\lambda}{2}(J-\alpha)^{2}+\frac{\gamma}{2}\left(e^{\frac{\mu_{1}}{2}\left(I_{C}-3\right)}-1\right),
$$

where the rightmost term is the novel one. Following the approach of Section 3.2, the energy can be stabilized by setting $\alpha=$ $1+\frac{\mu_{0}+\gamma \mu_{1}}{\lambda}$. The PK1 then expands to

$$
\mathbf{P}_{\text {Fung }}(\mathbf{F})=\mu_{0} \mathbf{F}+\lambda \frac{\partial J}{\partial \mathbf{F}}(J-\alpha)+\gamma \mu_{1} e^{\frac{\mu_{1}}{2}\left(I_{C}-3\right)} \mathbf{F} .
$$

The unfolded, assembled Hessian can then be written as

$$
\begin{aligned}
\check{\mathbf{A}}_{\text {Fung }}= & \left(\mu_{0}+\gamma \mu_{1} e^{\frac{\mu_{1}}{2}\left(I_{C}-3\right)}\right) \mathbf{I}+\lambda \check{\mathbf{g}} \check{g}^{T} \\
& +\lambda(J-\alpha) \check{\mathbf{H}}+2 \gamma \mu_{1}^{2} e^{\frac{\mu_{1}}{2}\left(I_{C}-3\right)} \check{\mathbf{f}}^{T}{ }^{T} .
\end{aligned}
$$

The following constants change in the eigenanalysis:

$$
\eta=\frac{2 \gamma \mu_{1}^{2} e^{\frac{\mu_{1}}{2}\left(I_{C}-3\right)}}{\lambda(J-\alpha)} \quad \rho=\frac{1}{J-\alpha} \quad \mu_{T}=\left(\mu_{0}+\gamma \mu_{1} e^{\frac{\mu_{1}}{2}\left(I_{C}-3\right)}\right) .
$$

Arruda-Boyce: The hardening term can also be represented with a polynomial using the model of Arruda and Boyce (1993):

$$
\Psi_{\mathrm{AB}}=\sum_{i=1}^{n} a_{i}\left(I_{C}^{i}-3^{i}\right) .
$$

We use the three-term expansion for illustrative purposes, shift the constants to maintain consistency with the Neo-Hookean parameters, and arrive at the following energy:

$$
\Psi_{\mathrm{AB}}=\frac{\mu}{2}\left(I_{C}-3\right)+\frac{\mu \beta_{1}}{4}\left(I_{C}^{2}-9\right)+\frac{\mu \beta_{2}}{6}\left(I_{C}^{3}-27\right)+\frac{\lambda}{2}(J-\alpha)^{2} .
$$

The energy is rest stable when $\alpha=1+\frac{\mu}{\lambda}\left(1+3 \beta_{1}+9 \beta_{2}\right)$, leading to a PK1 of

$$
\mathbf{P}_{\mathrm{AB}}(\mathbf{F})=\mu\left(1+\beta_{1} I_{C}+\beta_{2} I_{C}^{2}\right) \mathbf{F}+\lambda \frac{\partial J}{\partial \mathbf{F}}(J-\alpha)
$$

and a Hessian of

$$
\begin{aligned}
\check{\mathbf{A}}_{\mathrm{AB}}= & \mu\left(1+\beta_{1} I_{C}+\beta_{2} I_{C}^{2}\right) \mathbf{I}+\lambda \check{\mathbf{g}} \check{\mathbf{g}}^{T} \\
& +\lambda(J-\alpha) \check{\mathbf{H}}+2 \mu\left(\beta_{1}+2 \beta_{2} I_{C}\right) \check{\mathbf{f}}^{T} .
\end{aligned}
$$

This system leads to the following constants in the eigenanalysis:

$$
\eta=\frac{2 \mu\left(\beta_{1}+2 \beta_{2} I_{C}\right)}{\lambda(J-\alpha)} \quad \rho=\frac{1}{J-\alpha} \quad \mu_{T}=\mu\left(1+\beta_{1} I_{C}+\beta_{2} I_{C}^{2}\right) .
$$

\section{RESULTS}

We evaluate the robustness of our model across a variety of scenarios. All quasi-static examples use a (potentially nonmanifold (Mitchell et al. 2015)) hexahedral mesh with eight-point quadrature. To show the agnosticism of our energy to the topology and integration scheme, we also include a dynamic example using linear tetrahedra. Quasi-static simulations are performed with a standard Newton solver augmented with a line search, and linear systems are solved using ViennaCL's Conjugate Gradient implementation running on OpenCL (Rupp et al. 2010). All Newton solves were run until the absolute $L_{2}$-norm of the force residual was less than $1 e^{-2}$. After normalizing by the number of degrees of freedom, we are essentially solving to single precision. All simulations were performed with a 2.3GHz Intel Xeon E5-2699 on eight cores, 118GB of RAM, and an NVIDIA Quadro M6000 GPU.

Stretch Test. We stretch a cylinder composed of 306,406 hexahedra to 3.4 times its original length by translating hardconstrained vertices on two opposing faces. We compare to three models:

- The corotational energy $\Psi_{\mathrm{CR}}$ from Irving et al. (2004)

- The "fixed" corotational $\Psi_{\text {FixCR }}$ of Stomakhin et al. (2012)

- The original Neo-Hookean energy $\Psi_{\mathrm{Neo}}$, but "filtered" using Teran et al. (2005).

In all cases, we call the original implementations in the PhysBAM codebase (Dubey et al. 2011), which one of the original authors has generously provided for us. The default force-filtering threshold $\sigma_{f}=0.25$ and Hessian projection threshold $\epsilon_{p}=-1 e^{-4}$ from the code were used. We show results for an extreme Poisson's ratio of $v=0.49$ in Figure 5. A wide range of additional $v$, as well as comparisons to the $C_{2}$ model from Stomakhin et al. (2012) and the StVK 

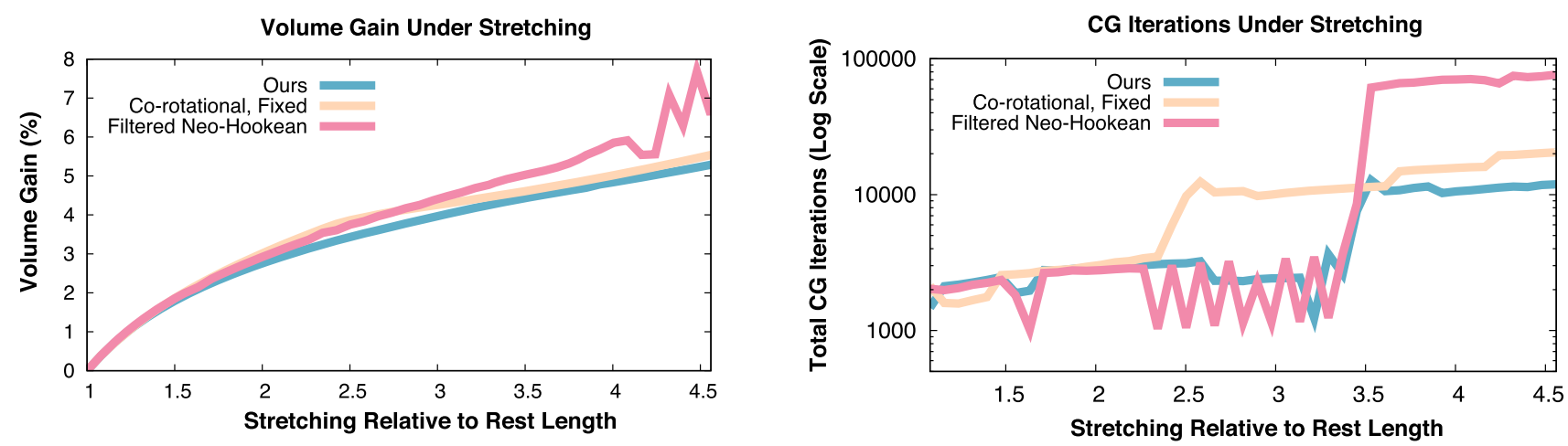

Fig. 6. In the left graph, our model exhibits the best volume preservation under stretching. In the right graph, our CG iteration count is competitive with the other models, and under large stretches, our model consistently converges faster. Our model took an average of 100.64s per frame, filtered Neo-Hookean took 537.72s, and "fixed" corotational took $144.07 \mathrm{~s}$.

model from Kikuuwe et al. (2009), are shown in the supplemental materials.

We first examine volume preservation. In Figure 6, the corotational model loses over $83.0 \%$ of the original volume; linearizing the volume penalty is clearly inadequate for this case. The "fixed" corotational model only gains $4.5 \%$ volume, but the final shape contains highly unnatural crinkling. An analogous artifact is shown in Figure 6 of Stomakhin et al. (2012), and our analysis suggests that it stems from the linearization hidden in the $\frac{\mu}{2}\|\mathbf{F}-\mathbf{R}\|_{F}^{2}$ term. Filtered Neo-Hookean gains $4.9 \%$ volume, slightly more than the other model. Finally, our stable Neo-Hookean model gains $4.3 \%$ volume, which is the smallest of any of the models.

In order to compare computational performance, we use the total number of conjugate gradient (CG) iterations performed by the Newton solver at each time step. This is more instructive than the total Newton iterations in this case, because many of the solves in Figure 5 completed in fewer than three iterations. It also removes constant factors associated with low-level optimization that has been applied to various components of the code, e.g., the PhysBAM classes.

The "fixed" corotational model requires the most CG iterations, roughly 2.3 times the total CG iterations of our model for the stretch in Figure 5. This may be because at larger deformations, the inconsistent approximations applied to the $\log J$ term begin to conflict. On average, our stable Neo-Hookean model takes slightly more CG iterations than filtered Neo-Hookean, with 1.2 times the total CG iterations; the computational costs are virtually identical. Our model contains none of the artifacts from the "fixed" corotational model and preserves volume better than the filtered NeoHookean model.

The CG iteration counts for the original corotational model are not shown because they are an order of magnitude larger than the other models. Converging to the degenerate geometry in Figure 5 is computationally expensive. We also attempted to simulate the $C_{2}$ continuous Neo-Hookean model from Stomakhin et al. (2012). While the model converged fine for lower $v$ (see supplemental materials), we were unable to locate settings that did not diverge before completing the test for $v=0.49$.
Twist Test: We take a unit cube and subject it to large kinematic rotations by hard-constraining one face of the cube and rotating it twice by $\pi / 2$ radians. Production quasi-static flesh simulations often undergo rotations of this magnitude, so it is critical that models remain robust under these conditions. The cube is discretized into $15^{3}$ hexahedra, and $v=0.49$. Our model finds the correct twisted shape, where the solves respectively took 12 and 16 Newton iterations, and 3,278 and 4,956 CG iterations (Figure 9).

The "fixed" corotational model is also able to robustly resolve both rotations in 11 and 15 Newton iterations and 2,940 and 4,348 CG iterations. The corotational model resolves the $\pi / 2$ rotation but then collapses. For the $\pi$ rotation, 223 Newton iterations were needed; resolving collapsed configurations is again costly. Collisions were disabled, so the results involve purely the material model. Filtered Neo-Hookean yields unusable results. The solve stalls when the line search is enabled and diverges when it is disabled.

Scramble. In Figure 3, we perform a "scramble" test similar to those in Teran et al. (2005) and Stomakin et al. (2012) by randomly placing the vertices of a unit cube within a cube of twice the rest volume. To separate the behavior of our model from that of massregularization, we perform a quasi-static solve. We pin four cube corners in order to constrain the rigid modes. As shown in the supplemental video, our model successfully recovers the rest pose.

Torso. In Figure 1, we perform a quasi-static flesh simulation of arms and a torso that are driven kinematically by a set of internal bones. The bones are transformed by the skinning of a production rig, which often results in nonrigid transforms and intersecting bones. Like the corotational model, our model is robust to these noisy inputs. However, our model produces realistic bulges and folds, while the corotational model produces artifacts and collapses the shoulder entirely. Increasing $v$ only worsens the artifact; the parameter accomplishes the opposite of its stated purpose. In Figure 10, we compare our model to an StVK model with a compression resistance term (Kikuuwe et al. 2009). The StVK model fails to capture the fleshy profile of our model.

Collisions are enabled in this simulation and handled with a reference map-based penalty force (Hirota et al. 2001; Irving et al. 

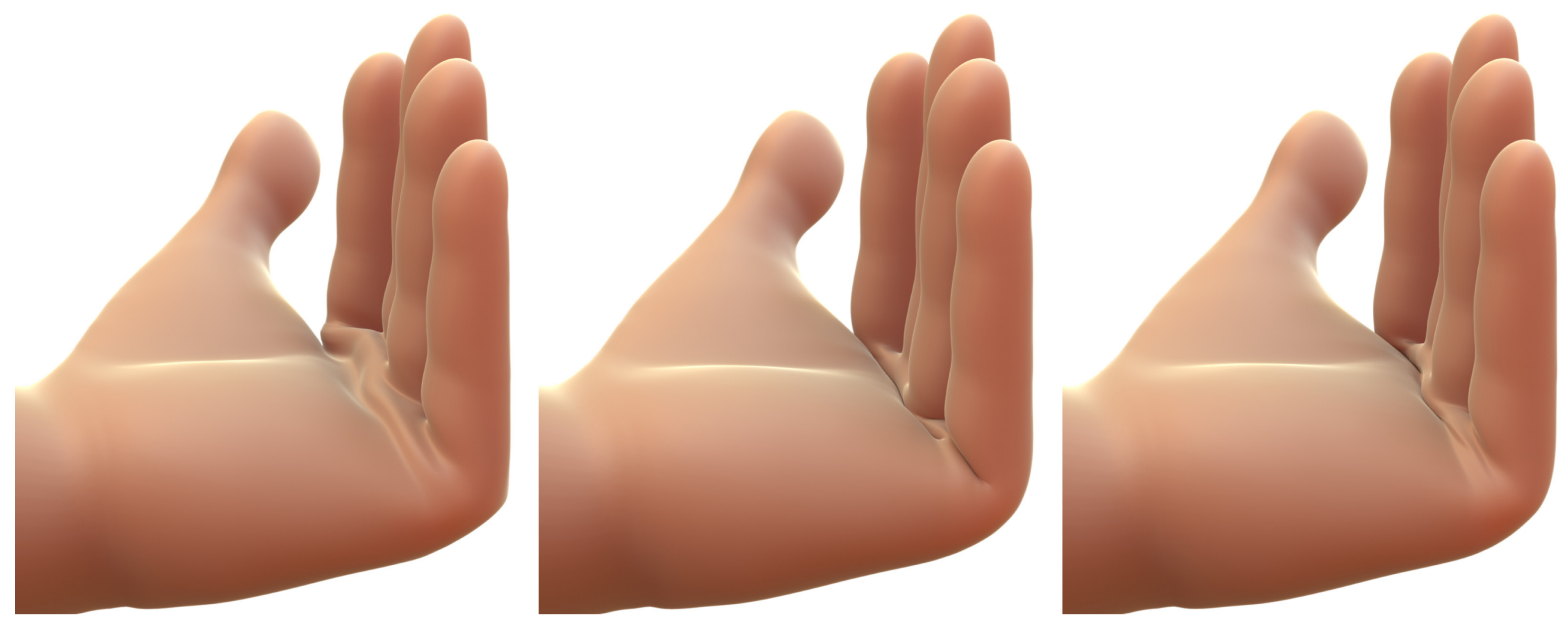

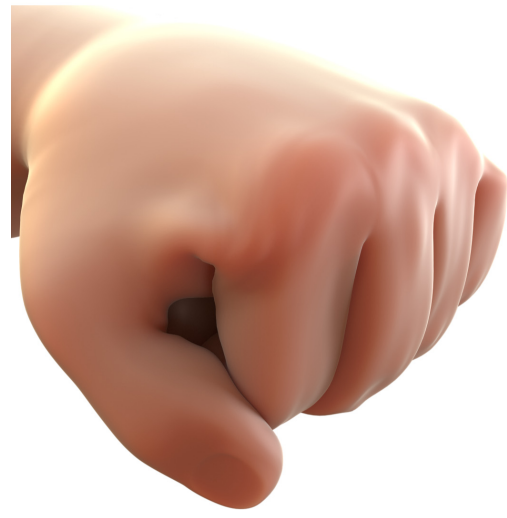

(a) Ours, $v=0.488$

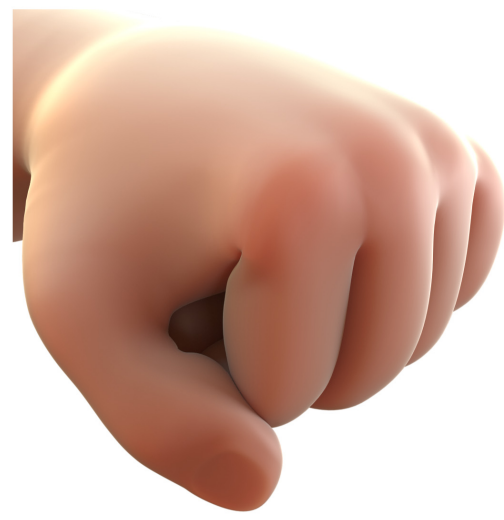

(b) Co-rotational, $v=0.2$

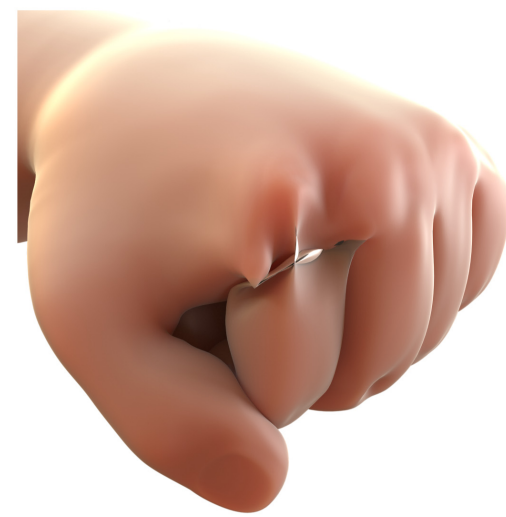

(c) Co-rotational, $v=0.488$

Fig. 7. Top: Our model captures the bulge in the palm at the base of the fingers. Bottom: Our model captures the bulge in the webbing between the pointer finger and the thumb. At $v=0.2$, corotational elasticity resembles contact-aware skinning. Increasing to $v=0.488$ only introduces artifacts and fails to capture the features of our model. The simulation is driven by 24 kinematic bones in the hand and the forearm, and the mesh consists of 41,050 hexahedra and 142,995 degrees of freedom. Stable Neo-Hookean took an average of 15.9 Newton iterations, 9,486.7 CG iterations, and 53.8 seconds. With corotational at $v=0.2$, the average is 13.1 Newton iterations, $2,471.9 \mathrm{CG}$ iterations, and 44.0 seconds. With corotational at $v=0.488$, the simulation did not complete. cDisney/Pixar.

2004). Starting from a surface point sampling, a spatial grid acceleration structure (McAdams et al. 2011) is used to locate sample points in a tetrahedralization of the hexahedral mesh. If a sample point collides with a tetrahedron, it is mapped to the reference domain, where the closest surface point is computed. The surface point and the original surface sample are mapped back to the deformed domain and define a penalty spring. Due to the two linear maps, this target position is not always the closest point on the deformed surface mesh. If clean and conforming contacts are desired, an additional local optimization is performed on the deformed surface mesh that iteratively searches for closer points. This process typically converges in a single iteration.

Hand. In Figures 7 and 8, we simulate the flesh on a hand and forearm. As with the torso, bones drive the simulation, but their transforms come from a skinning that is noisy and contains difficult stretches and intersections. Similar to McAdams et al. (2011), the simulation both cleans up skinning artifacts around difficult joints and adds physics-based details that were missing in the original rig.

The corotational model consistently collapses regions where volume should be preserved, such as wrinkles in the palm of the hand or folds in the webbing between the thumb and pointer finger. As with the torso example, increasing $v$ can worsen artifacts and in some cases causes the Newton solve to diverge.

Dynamics. In Figure 11, we show our material model undergoing dynamic, extreme collisions. Despite the majority of the model being squashed to almost zero volume, our elastic energy allows it to recover. The simulation uses a tetrahedral mesh and shows that our model is agnostic to element type and quadrature scheme.

\section{CONCLUSIONS AND FUTURE WORK}

We have designed a new hyperelastic energy that requires no special machinery to remain stable under large deformations, 

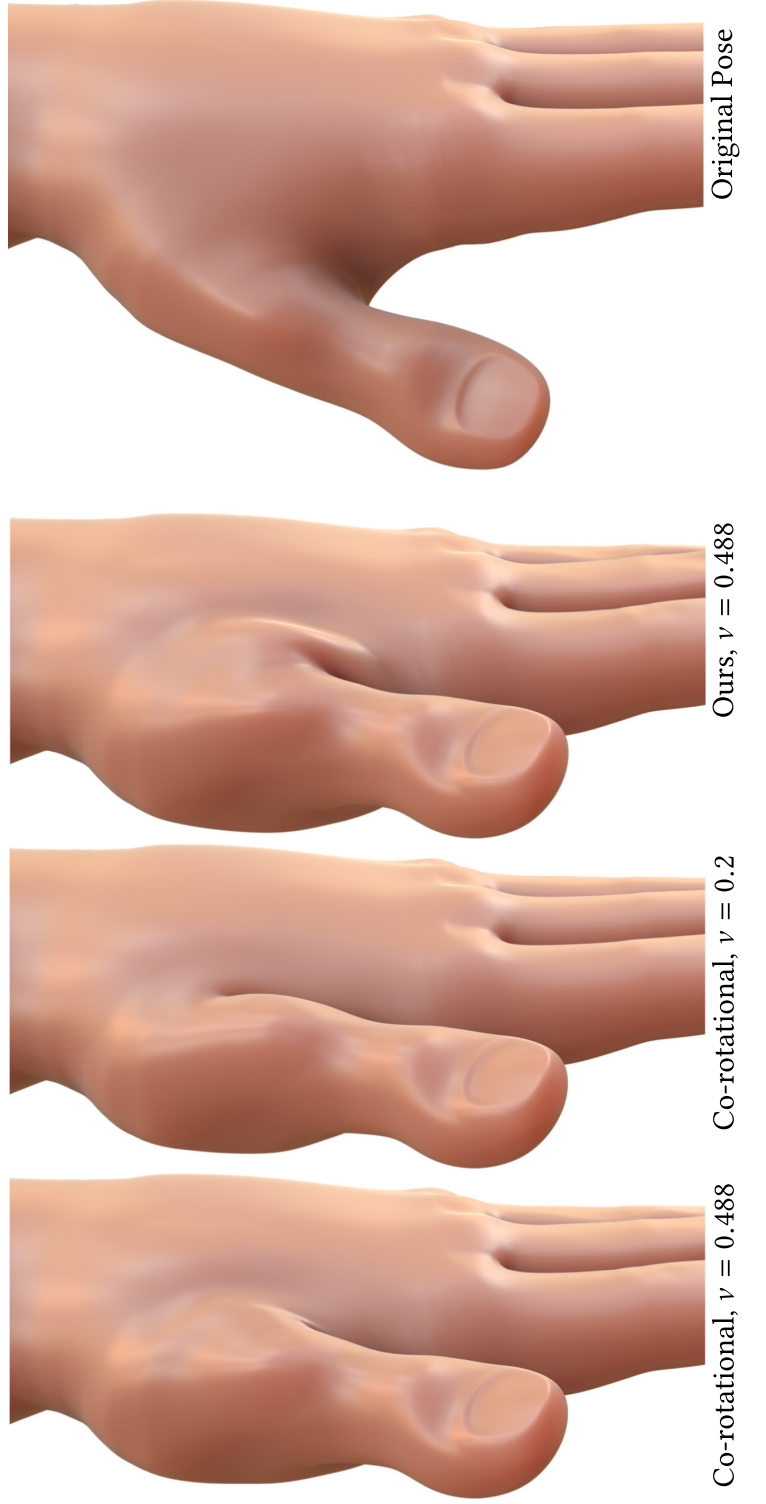

Fig. 8. Top: The thumb at its original rest pose. Top, middle: When pinched, the flesh between the thumb and pointer bulges with our model set to $v=0.488$. Bottom, middle: With the corotational model, there is no feature at $v=0.2$. Bottom: Only slight bulging appears in the corotational model at $v=0.488$. The settings and timings are the same as Figure 7. ๑Disney/Pixar.

including inversions. We are able to derive closed-form expressions for the eigendecompositions of all its components. The sum of these components also possesses a special structure that allows us to write simple expressions for the eigensystem. Together, these findings allow our energy to be used in Newton-based implicit schemes that use conjugate gradient solvers.

We have established a hierarchy of Neo-Hookean approximations. The corotational elasticity model is the simplest, as it applies

\begin{tabular}{|c|ccc|}
\hline $\begin{array}{c}\text { Twist } \\
\text { Angle }\end{array}$ & $\begin{array}{c}\text { Stable } \\
\text { Neo-Hookean }\end{array}$ & $\begin{array}{c}\text { Filtered } \\
\text { Neo-Hookean }\end{array}$ & $\begin{array}{c}\text { Fixed } \\
\text { Co-rotational }\end{array}$ \\
\hline$\frac{\pi}{2}$ & & & \\
\hline$\pi$
\end{tabular}

Fig. 9. The top face of the cube undergoes two successive $\pi / 2$ rotations. Stable Neo-Hookean and "fixed" corotational resolve the twist robustly. Corotational has trouble with the second rotation, and filtered NeoHookean is unable to resolve the shape. The cube is discretized with $15^{3}$ hexahedral elements and the material models are simulated with $v=0.49$.
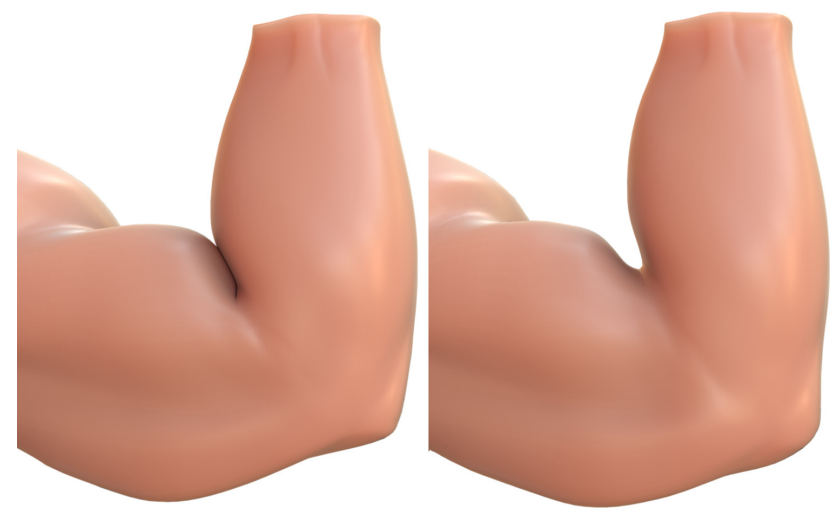

Fig. 10. Left: A bicep under an imposed elbow flexion simulated with our stable Neo-Hookean model $(v=0.488)$. An average time step took 13.7 Newton iterations, 5,860 conjugate gradient (CG) iterations, and 25.6 seconds. Right: The same pose with StVK and a compression resistance term $\left(v=0.488, \kappa=8 \times 10^{8}\right)$. The StVK model fails to capture the fleshy silhouette we observe with our model, and instead leaves a gap between the bicep and forearm. An average time step with this model took 32 Newton iterations, 14,446 CG iterations, and 140.3 seconds. @Disney/Pixar.

the $\log J \approx \operatorname{tr}(\mathrm{S}-\mathrm{I})$ linearization to both Neo-Hookean terms, followed by the model of Stomakhin et al. (2012), which only applies it to one. Our energy instead consistently uses $\log J \approx(J-1)$ everywhere. This critical difference allows volume to be measured accurately under large deformation, and improves both the stability and quality of the simulation. The $\log J \approx(J-1)$ approximation is a first-order truncation, so one direction for future work is to determine the benefits of higher-order truncations.

In Section 4, we performed a complete eigenanalysis of the first stress invariant $I_{C}$ and the volume measure $J$. Our analysis of $J$ also constitutes an analysis of the third stress invariant, $I I I_{C}=J^{2}$. These results can be used to analyze any energy that can be written in terms of $I_{C}$ and $I I I_{C}$, as we show with the Fung and ArrudaBoyce models in Section 5.3. An eigenanalysis of the second invariant, $I I_{C}=\operatorname{tr}\left(C^{T} C\right)$, is still missing, and could lead to a better understanding of materials such as the Mooney-Rivlin and Verdona models. 


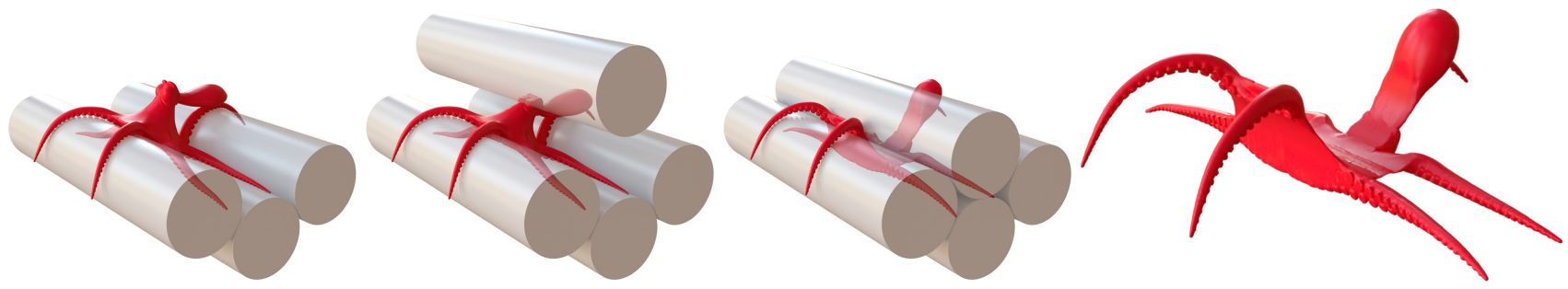

Fig. 11. We dynamically drop an octopus containing 394,268 tetrahedra and 278,991 degrees of freedom with $v=0.46$ onto three cylinders. We squash it with another cylinder, but it recovers as soon as the cylinder is removed. For clarity, the cylinders are visualized with partial transparency. On the right is a closeup of the most squashed state. The simulation took 10 semi-implicit (Baraff and Witkin 1998) substeps per frame to resolve the collisions, and each frame took an average of 20.8 seconds. $\odot$ Disney/Pixar.

Finally, a better understanding of how Hessian indefiniteness impacts Newton convergence is needed. Allowing a slight amount of indefiniteness to enter into the global Hessian can sometimes accelerate Newton convergence, so a better understanding of this phenomenon could yield significant practical benefits.

\section{APPENDIXES}

\section{A VOLUME HESSIAN EIGENVECTORS}

The first three eigenvectors, $\varepsilon_{0} \ldots 2$, are specified by Equations (39) and (40). The remaining six, $\varepsilon_{3} \ldots 8$, are of the form

$$
\mathrm{Q}_{k}=\frac{1}{\sqrt{2}} \mathrm{UD}_{k} \mathbf{V}^{T} \text {. }
$$

The only differences are in the $\mathrm{D}_{i}$ matrices, which are

$$
\begin{array}{ll}
\mathbf{D}_{3}=\left[\begin{array}{rrr}
0 & 0 & 0 \\
0 & 0 & 1 \\
0 & -1 & 0
\end{array}\right] & \mathbf{D}_{6}=\left[\begin{array}{lll}
0 & 0 & 0 \\
0 & 0 & 1 \\
0 & 1 & 0
\end{array}\right] \\
\mathbf{D}_{4}=\left[\begin{array}{rrr}
0 & 0 & 1 \\
0 & 0 & 0 \\
-1 & 0 & 0
\end{array}\right] & \mathbf{D}_{7}=\left[\begin{array}{lll}
0 & 0 & 1 \\
0 & 0 & 0 \\
1 & 0 & 0
\end{array}\right] \\
\mathbf{D}_{5}=\left[\begin{array}{rrr}
0 & 1 & 0 \\
-1 & 0 & 0 \\
0 & 0 & 0
\end{array}\right] & \mathbf{D}_{8}=\left[\begin{array}{lll}
0 & 1 & 0 \\
1 & 0 & 0 \\
0 & 0 & 0
\end{array}\right] .
\end{array}
$$

\section{B ORTHOGONALITY TO PSEUDO-CROSS-PRODUCTS}

Theorem B.1. If $\mathbf{B} \in \mathbb{R}^{3 \times 3}$ can be diagonalized using the $\mathbf{U}$ and V from $\mathbf{F}$, then $\operatorname{vec}(\mathbf{B})=\breve{b}$ is orthogonal to all six pseudo-crossproduct eigenvectors in Equation (62).

Proof. We denote the eigenvectors in flattened form as $\operatorname{vec}\left(Q_{k}\right)=\check{\mathbf{q}}_{k}$. If we can show that $\check{\mathbf{q}}_{k}^{T} \check{\mathrm{b}}^{2}=0$, then we are done. First, we observe that $\check{\mathbf{q}}_{k}^{T} \check{\mathbf{b}}=\operatorname{tr}\left(\mathbf{Q}_{k}^{T} \mathbf{B}\right)$. Next, we use the assumption that $\mathbf{B}$ can be diagonalized: $\mathbf{B}=\mathbf{U} \Sigma_{\mathbf{B}} \mathbf{V}^{T}$. Since $\mathbf{Q}_{k}=\mathbf{U D}_{k} \mathbf{V}^{T}$, we have $\operatorname{tr}\left(\mathbf{Q}_{k}^{T} \mathbf{B}\right)=\operatorname{tr}\left(\mathbf{D}_{k}^{T} \Sigma_{\mathbf{B}}\right)=0$. Finally, we observe that $\mathbf{D}_{k}$ are hollow matrices; i.e., they are zero along their diagonals. The trace of a diagonal times a hollow matrix is zero. Thus, $\check{\mathbf{q}}_{k}^{T} \check{\mathbf{b}} \equiv \operatorname{tr}\left(Q_{k}^{T} \mathbf{B}\right) \equiv$ $\operatorname{tr}\left(\mathbf{D}_{k}^{T} \Sigma_{\mathbf{B}}\right)=0$.

Corollary B.2. The vector $\check{\mathrm{g}}$ is orthogonal to all six pseudocross-product eigenvectors.
Proof. The vector $\check{\mathrm{g}}$ is generated from the matrix $\frac{\partial J}{\partial \mathrm{F}}$, i.e., $\operatorname{vec}\left(\frac{\partial J}{\partial \mathrm{F}}\right)=\check{\mathrm{g}}$. This matrix can be diagonalized as

$$
\frac{\partial J}{\partial \mathrm{F}}=\mathbf{U}\left[\begin{array}{rrr}
\sigma_{1} \sigma_{2} & 0 & 0 \\
0 & \sigma_{0} \sigma_{2} & 0 \\
0 & 0 & \sigma_{0} \sigma_{1}
\end{array}\right] \mathbf{V}^{T} .
$$

By the preceding theorem, $\check{\mathrm{g}}$ is orthogonal.

\section{COROTATIONAL AS LINEARIZED NEO-HOOKEAN}

Lemma C.1. $\operatorname{tr}(\mathrm{S}-\mathrm{I})$ is a linearization of $\log J$.

Proof. Given the polar decomposition F = RS (Table 1), we have $J=\operatorname{det} \mathrm{F}=\operatorname{det} \mathrm{S}$. By applying the identity $\operatorname{det} \mathrm{S}=e^{\operatorname{tr}(\log \mathrm{S})}$, we obtain $\log J=\operatorname{tr}(\log \mathrm{S})$. We then compute the Taylor expansion about $\mathrm{S}=$ I,

$$
\log \mathrm{S}=(\mathrm{S}-\mathrm{I})-\frac{1}{2}(\mathrm{~S}-\mathrm{I})^{2}+\frac{1}{3}(\mathrm{~S}-\mathrm{I})^{3} \ldots,
$$

and employ its linear term to conclude: $\log J \approx \operatorname{tr}(\mathrm{S}-\mathrm{I})$.

Theorem C.2. $\mu / 2|| \mathbf{F}-\mathbf{R} \|_{F}^{2}$ is a linearization of $\mu / 2\left(I_{C}-3\right)-$ $\mu \log J$.

Proof. This follows from an algebraic manipulation:

$$
\begin{aligned}
\frac{\mu}{2}\left(I_{C}-3\right)-\mu \log J & =\frac{\mu}{2}\left(\|\mathbf{F}\|_{F}^{2}-\operatorname{tr} \mathbf{I}\right)-\mu \log J \\
\text { (applying Lemma C.1) } & \approx \frac{\mu}{2}\left(\|\mathbf{F}\|_{F}^{2}-\operatorname{tr} \mathbf{I}\right)-\mu \operatorname{tr}(\mathrm{S}-\mathbf{I}) \\
& =\frac{\mu}{2}\left(\|\mathbf{F}\|_{F}^{2}+\operatorname{tr} \mathbf{I}-2 \operatorname{trS}\right) \\
& =\frac{\mu}{2}\left(\|\mathbf{F}\|_{F}^{2}+\|\mathbf{R}\|_{F}^{2}-2 \operatorname{tr}\left(\mathbf{R}^{T} \mathbf{F}\right)\right) \\
& =\frac{\mu}{2}\|\mathbf{F}-\mathbf{R}\|_{F}^{2} .
\end{aligned}
$$

Therefore: $\frac{\mu}{2}\left(I_{C}-3\right)-\mu \log J \approx \frac{\mu}{2}\|\mathbf{F}-\mathbf{R}\|_{F}^{2}$.

Corollary C.3. $\lambda / 2 \operatorname{tr}^{2}(\mathrm{~S}-\mathrm{I})$ is a linearization of $\lambda / 2(\log J)^{2}$.

Proof. We can reuse Lemma C.1 and the linearization appears immediately.

\section{EIGENSYSTEM WHEN $v \geq 0.4$}

If fleshy material with $v \geq 0.4$ is being simulated, the regularized origin barrier can be discarded, and the simpler eigensystem from that results from Equation (13) can be used instead. The Hessian is then

$$
\check{\mathbf{A}}=\mu \mathbf{I}+\lambda \check{\mathbf{g}} \check{g}^{T}+\lambda(J-\alpha) \check{\mathbf{H}},
$$


which is only a rank-one updated system. The deflation argument from Section 4.6 still applies, and the rank-one updated cubic is instead

$$
\bar{\varepsilon}^{3}-\rho\|\grave{\mathbf{g}}\|_{2}^{2} \bar{\varepsilon}^{2}-(1+2 \rho J)\|\mathbf{F}\|_{F}^{2} \bar{\varepsilon}-(2+3 \rho J) J=0,
$$

where $\rho=(J-\alpha)^{-1}$. The diagonal entries in the eigenvector $\mathbf{Q}_{k}=$ $\frac{1}{q_{k}} \mathbf{U D}_{k} \mathbf{V}^{T}$ can be written as

$$
\begin{aligned}
\mathbf{D}_{k} & =\left[\begin{array}{rrr}
\alpha\left(\sigma_{0} \sigma_{2}+\sigma_{1} \bar{\varepsilon}_{k}\right) & 0 & 0 \\
0 & \alpha\left(\sigma_{1} \sigma_{2}+\sigma_{0} \bar{\varepsilon}_{k}\right) & 0 \\
0 & 0 & \bar{\varepsilon}_{k}^{2}-\beta \sigma_{2}^{2}
\end{array}\right] \\
\alpha & =1+\rho J \\
\beta & =\alpha+\rho\left(J+\left(\sigma_{0}^{2}+\sigma_{1}^{2}\right) \bar{\varepsilon}_{i}\right) .
\end{aligned}
$$

Note that for the case of $\rho=0$, Equation (40) is recovered. The final eigenvalues are now the same as in Equations (45) to (48), except that the roots of Equation (66) are used instead, and $\mu_{T}=\mu$.

\section{ACKNOWLEDGMENTS}

We would like to thank David Eberle and Audrey Wong for their last-minute help getting the tetrahedral octopus simulation from Figure 11 pushed through Presto and Fizt. We would also like to thank Jonathan Page for providing both the bone geometry and finger animation in Figure 7, Bill Wise for providing the geometry and animation for the torso example in Figure 1, and Rick Sayre for approving the use of the torso asset. Thanks also to Tony DeRose and Doug James for their feedback on early drafts of this work.

\section{REFERENCES}

Ellen M. Arruda and Mary C. Boyce. 1993. A three-dimensional constitutive model for the large stretch behavior of rubber elastic materials. Fournal of the Mechanics and Physics of Solids 41, 2 (1993), 389-412.

David Baraff and Andrew Witkin. 1998. Large steps in cloth simulation. In Proceedings of the 25th Annual Conference on Computer Graphics and Interactive Techniques (SIGGRAPH'98). 43-54.

Adam W. Bargteil and Elaine Cohen. 2014. Animation of deformable bodies with quadratic bézier finite elements. ACM Trans. Graph. 33, 3, Article 27 (June 2014), 10 pages.

Jan Bender, Matthias Müller, and Miles Macklin. 2015. Position-based simulation methods in computer graphics. In Eurographics Tutorials. Eurographics Association.

Silvia S. Blemker, Peter M. Pinsky, and Scott L. Delp. 2005. A 3D model of muscle reveals the causes of nonuniform strains in the biceps brachii. F. Biomechanics 38 , 4 (2005), 657-665.

Javier Bonet and Richard D. Wood. 2008. Nonlinear Continuum Mechanics for Finite Element Analysis. Cambridge University Press.

Sofien Bouaziz, Sebastian Martin, Tiantian Liu, Ladislav Kavan, and Mark Pauly. 2014. Projective dynamics: Fusing constraint projections for fast simulation. ACM Trans. Graph. 33, 4, Article 154 (July 2014), 11 pages.

Allan F. Bower. 2009. Applied Mechanics of Solids. CRC Press.

James R. Bunch, Christopher P. Nielsen, and Danny C. Sorensen. 1978. Rank-one modification of the symmetric eigenproblem. Numer. Math. 31, 1 (1978), 31-48.

Isaac Chao, Ulrich Pinkall, Patrick Sanan, and Peter Schröder. 2010. A simple geometric model for elastic deformations. ACM Trans. Graph. 29, 4, Article 38 (2010), 6 pages.

Oscar Civit-Flores and Antonio Susín. 2014. Robust treatment of degenerate elements in interactive corotational FEM simulations. Comput. Graph. Forum 33, 6 (2014), 298-309.

Pradeep Dubey, Pat Hanrahan, Ronald Fedkiw, Michael Lentine, and Craig Schroeder. 2011. PhysBAM: Physically based simulation. In ACM SIGGRAPH Courses. Article 10, 22 pages.

Yuan-cheng Fung. 2013. Biomechanics: Mechanical Properties of Living Tissues Springer Science \& Business Media.

Joachim Georgii and Rüdiger Westermann. 2008. Corotated finite elements made fast and stable. In Proceedings of the Fifth Workshop on Virtual Reality Interaction and Physical Simulation (VRIPHYS'08). 11-19.
Gene H. Golub and Charles F. Van Loan. 2012. Matrix Computations. Vol. 3. JHU Press. George Neville Greaves, A. L. Greer, R. S. Lakes, and T. Rouxel. 2011. Poisson's ratio and modern materials. Nat. Mater. 10, 11 (2011), 823-837.

Nicholas J. Higham. 2008. Functions of Matrices: Theory and Computation. SIAM.

Gentaro Hirota, Susan Fisher, A. State, Chris Lee, and Henry Fuchs. 2001. An implicit finite element method for elastic solids in contact. In Proceedings of the Fourteenth Conference on Computer Animation (Computer Animation'01). 136-254.

Geoffrey Irving, Joseph Teran, and Ronald Fedkiw. 2004. Invertible finite elements for robust simulation of large deformation. In ACM SIGGRAPH/Eurographics Symposium on Computer Animation (SCA'04). 131-140.

Michael A. Jenkins and Joseph F. Traub. 1970. A three-stage variable-shift iteration for polynomial zeros and its relation to generalized rayleigh iteration. Numer. Math. 14, 3 (1970), 252-263.

Ryan Kautzman, Jiayi Chong, and Patrick Coleman. 2012. Stable, art-directable skin and flesh using biphasic materials. In ACM SIGGRAPH Talks.

Ryo Kikuuwe, Hiroaki Tabuchi, and Motoji Yamamoto. 2009. An edge-based computationally efficient formulation of Saint Venant-Kirchhoff tetrahedral finite elements. ACM Trans. Graph. 28, 1, Article 8 (2009), 13 pages.

Allen Knutson and Terence Tao. 2001. Honeycombs and sums of hermitian matrices. Notices Amer. Math. Soc 48, 2 (2001), 175-186.

Tamara G. Kolda and Brett W. Bader. 2009. Tensor decompositions and applications. SIAM Rev. 51, 3 (2009), 455-500.

Tiantian Liu, Ming Gao, Lifeng Zhu, Eftychios Sifakis, and Ladislav Kavan. 2016. Fast and robust inversion-free shape manipulation. Comput. Graph. Forum 35, 2 (2016), 1-11.

Sebastian Martin, Bernhard Thomaszewski, Eitan Grinspun, and Markus Gross. 2011. Example-based elastic materials. ACM Trans. Graph. 30, 4, Article 72 (July 2011), 8 pages.

Aleka McAdams, Yongning Zhu, Andrew Selle, Mark Empey, Rasmus Tamstorf, Joseph Teran, and Eftychios Sifakis. 2011. Efficient elasticity for character skinning with contact and collisions. ACM Trans. Graph. 30, 4, Article 37 (July 2011), 12 pages.

Nathan Mitchell, Mridul Aanjaneya, Rajsekhar Setaluri, and Eftychios Sifakis. 2015 Non-manifold level sets: A multivalued implicit surface representation with applications to self-collision processing. ACM Trans. Graph. 34, 6, Article 247 (Oct. 2015), 9 pages.

Melvin Mooney. 1940. A theory of large elastic deformation. F. Appl. Phys. 11, 9 (1940) 582-592.

Matthias Müller, Julie Dorsey, Leonard McMillan, Robert Jagnow, and Barbara Cutler. 2002. Stable real-time deformations. In ACM SIGGRAPH/Eurographics Symposium on Computer Animation (SCA'02). 49-54.

Matthias Müller, Jos Stam, Doug James, and Nils Thürey. 2008. Real time physics. In ACM SIGGRAPH Classes. Article 88, 90 pages.

Rahul Narain, Matthew Overby, and George E. Brown. 2016. ADMM $\supseteq$ projective dynamics: Fast simulation of general constitutive models. In Proceedings of the ACM SIGGRAPH/Eurographics Symposium on Computer Animation (SCA'16). 2128.

Matthieu Nesme, Paul G. Kry, Lenka Jeřábková, and François Faure. 2009. Preserving topology and elasticity for embedded deformable models. ACM Trans. Graph. 28, 3, Article 52 (July 2009), 9 pages.

Raymond W. Ogden. 1997. Non-linear Elastic Deformations. Dover Publications.

Zherong Pan, Hujun Bao, and Jin Huang. 2015. Subspace dynamic simulation using rotation-strain coordinates. ACM Trans. Graph. 34, 6, Article 242 (2015) 12 pages.

Ronald Rivlin. 1948. Large elastic deformations of isotropic materials. IV. Further developments of the general theory. Philos. Trans. Roy. Soc. London A: Math. Phys. Eng. Sci. 241, 835 (1948), 379-397.

Karl Rupp, Florian Rudolf, and Josef Weinbub. 2010. ViennaCL - A high level linear algebra library for GPUs and multi-core CPUs. In International Workshop on GPUs and Scientific Applications. 51-56.

Ruediger Schmedding and Matthias Teschner. 2008. Inversion handling for stable deformable modeling. Vis. Comput. 24, 7 (2008), 625-633.

Christian Schüller, Ladislav Kavan, Daniele Panozzo, and Olga Sorkine-Hornung. 2013. Locally injective mappings. In Eurographics/ACMSIGGRAPH Symposium on Geometry Proceedings (SGP'13). 125-135.

Eftychios Sifakis and Jernej Barbic. 2012. FEM simulation of 3D deformable solids: A practitioner's guide to theory, discretization and model reduction. In ACM SIGGRAPH Courses. Article 20, 50 pages.

James G. Simmonds. 2012. A Brief on Tensor Analysis. Springer Science \& Business Media.

Alexey Stomakhin, Russell Howes, Craig Schroeder, and Joseph M. Teran. 2012. Energetically consistent invertible elasticity. In ACM SIGGRAPH/Eurographics Symposium on Computer Animation (SCA'12). 25-32.

Joseph Teran, Eftychios Sifakis, Geoffrey Irving, and Ronald Fedkiw. 2005. Robust quasistatic finite elements and flesh simulation. In ACM SIGGRAPH/Eurographics Symposium on Computer Animation (SCA'05). 181-190. 
Matthias Teschner, Bruno Heidelberger, Matthias Muller, and Markus Gross. 2004. A versatile and robust model for geometrically complex deformable solids. In Proceedings of Computer Graphics International (CGI'04). 312-319.

Christopher D. Twigg and Zoran Kačić-Alesić. 2010. Point cloud glue: Constraining simulations using the procrustes transform. In ACM SIGGRAPH/Eurographics Symposium on Computer Animation (SCA'10). 45-54.

Nobuyuki Umetani, Ryan Schmidt, and Jos Stam. 2014. Position-based elastic rods. In ACM SIGGRAPH/Eurographics Symposium on Computer Animation (SCA'14). 2130 .
Huamin Wang and Yin Yang. 2016. Descent methods for elastic body simulation on the GPU. ACM Trans. Graph. 35, 6, Article 212 (Nov. 2016), 10 pages.

Hongyi Xu, Funshing Sin, Yufeng Zhu, and Jernej Barbič. 2015. Nonlinear material design using principal stretches. ACM Trans. Graph. 34, 4, Article 75 (July 2015), 11 pages.

Received September 2017; accepted November 2017 